TRANSACTIONS OF THE

AMERICAN MATHEMATICAL SOCIETY

Volume 348, Number 8, August 1996

\title{
QUADRATIC FORMS FOR THE 1-D SEMILINEAR SCHRÖDINGER EQUATION
}

\author{
CARLOS E. KENIG, GUSTAVO PONCE, AND LUIS VEGA
}

\begin{abstract}
This paper is concerned with 1-D quadratic semilinear Schrödinger equations. We study local well posedness in classical Sobolev space $H^{s}$ of the associated initial value problem and periodic boundary value problem. Our main interest is to obtain the lowest value of $s$ which guarantees the desired local well posedness result. We prove that at least for the quadratic cases these values are negative and depend on the structure of the nonlinearity considered.
\end{abstract}

\section{INTRODUCTION}

This paper is concerned with the semilinear Schrödinger equation. Our purpose is to study the well posedness of the associated initial value problem (IVP) and periodic boundary value problem (pbvp) under low regularity of the data.

To measure this regularity we shall use the classical Sobolev spaces $H^{s}\left(\mathbb{R}^{n}\right)$ or $H^{s}\left(\mathbb{T}^{n}\right)$, and remark that our main interest is in the nonlinearities for which the Sobolev index $s$ can take negative values, i.e., $s<0$.

In this work we restrict ourselves to the one-dimensional case with quadratic homogeneous nonlinearities.

The IVP for the 1-D semilinear Schrödinger equation

$$
\left\{\begin{array}{l}
\partial_{t} u=i \partial_{x}^{2} u+N(u, \bar{u}), \quad x, t \in \mathbb{R}, \\
u(x, 0)=u_{0}(x)
\end{array}\right.
$$

as well as its higher dimensional version, has been extensively studied (see [C], $[\mathrm{CW}],[\mathrm{GV} 1],[\mathrm{K} 2],[\mathrm{T}]$ and references therein). (Here, we shall only consider the case where $N(\cdot, \cdot)$ is a polynomial.) In particular, T. Cazenave and F. Weissler $[\mathrm{CW}]$ and Y. Tsutsumi $[\mathrm{T}]$ have shown that for $u_{0} \in L^{2}(\mathbb{R})$ the IVP (1.1) is locally well posed for every polynomial $N$ of degree $\leq 5$, i.e.,

$$
N\left(z_{1}, z_{2}\right)=\sum_{|\alpha| \leq 5} a_{\alpha} z_{1}^{\alpha_{1}} z_{2}^{\alpha_{2}} .
$$

The proof of this result is based on the version of the Strichartz estimate [S] for the free Schrödinger group $\left\{e^{i t \Delta}\right\}_{-\infty}^{\infty}$ found in [GV2], which in the 1-D case affirms

Received by the editors May 17, 1995.

1991 Mathematics Subject Classification. Primary 35K22; Secondary 35P05.

Key words and phrases. Schrödinger equation, bilinear estimates, well-posedness.

C. E. Kenig and G. Ponce were supported by NSF grants. L. Vega was supported by a DGICYT grant. 
that

$$
\left(\int_{-\infty}^{\infty}\left\|e^{i t \partial_{x}^{2}} u_{0}\right\|_{L_{x}^{p}}^{r} d t\right)^{1 / r} \leq c\left\|u_{0}\right\|_{L^{2}}
$$

for $r, p \in[2, \infty]$ with $\frac{2}{r}=\frac{1}{2}-\frac{1}{p}$.

This local well posedness result depends on the degree of the nonlinearity $N(\cdot)$, and its proof does not distinguish any other structure on $N(\cdot)$.

We observe that for $N(\cdot)$ homogeneous of degree $k$, i.e., $|\alpha|=k$ in (1.2), it follows that if $u=u(x, t)$ solves (1.1) so does

$$
u_{\lambda}(x, t)=\lambda^{2 /(k-1)} u\left(\lambda x, \lambda^{2} t\right), \quad \text { for any } \lambda \in \mathbb{R},
$$

with data

$$
u_{\lambda}(x, 0)=\lambda^{2 /(k-1)} u_{0}(\lambda x)
$$

In particular, for $k=5$

$$
\left\|u_{\lambda}(0)\right\|_{L^{2}}=\left\|u_{0}\right\|_{L^{2}}, \quad \text { for any } \lambda \in \mathbb{R} .
$$

The scaling argument in (1.4)-(1.6) suggests that in this setting, 1-D, $N(\cdot)$ homogeneous of degree 5 and $u_{0} \in L^{2}(\mathbb{R})$, the result in [CW] should be optimal. This was proven in [BKPSV] for the nonlinearity $N\left(z_{1}, z_{2}\right)=-i \mu z_{1}^{3} z_{2}^{2}$, with $\mu>0$. Also this scaling argument hints that for lower nonlinearities $N(\cdot)$ one may expect local well posedness results in $H^{s}(\mathbb{R})$ with $s<0$. However no results in this direction were previously known.

As it was mentioned above, here we study the IVP (1.1) with nonlinearities

$$
N_{1}(u, \bar{u})=c_{1} u u, \quad N_{2}(u, \bar{u})=c_{2} u \bar{u} \quad \text { and } \quad N_{3}(u, \bar{u})=c_{3} \bar{u} \bar{u} .
$$

It will be proven that for the nonlinearities $N_{1}(\cdot)$ and $N_{3}(\cdot)$ the IVP (1.1) is locally well posed in $H^{s}(\mathbb{R})$, for $s>-3 / 4$, and that a similar result holds for $N_{2}(\cdot)$ in $H^{s}(\mathbb{R})$ with $s>-1 / 4$.

It is interesting to compare these results with those known for other evolution models.

For the IVP for the generalized Korteweg-de Vries

$$
\left\{\begin{array}{l}
\partial_{t} u+\partial_{x}^{3} u+\partial_{x}\left(u^{k+1}\right)=0, \quad x, t \in \mathbb{R}, \quad k=1,2, \ldots, \\
u(x, 0)=u_{0}(x)
\end{array}\right.
$$

we showed in [KPV1] that for $k \geq 4(1.8)$ is locally well posed in $H^{s}(\mathbb{R}), s \geq s(k)=$ $(k-4) / 2 k$, as the scaling argument suggests, and in [BKPSV] that these results are sharp. Also in [KPV1] we established similar local existence results for $k=2,3$ and $s(k)=1 / 4,1 / 12$ respectively. In [KPV3], for the $\mathrm{KdV}$ equation, $k=1$, we obtain local well posedness for $s>-3 / 4$ (see also [B]).

In a recent work $[\mathrm{D}] \mathrm{D}$. Dix has shown that the IVP for Burgers' equation

$$
\left\{\begin{array}{l}
\partial_{t} u-\partial_{x}^{2} u+\partial_{x}\left(u^{2}\right)=0, \quad x, t \in \mathbb{R} \\
u(x, 0)=u_{0}(x)
\end{array}\right.
$$

is ill-posed, more precisely, uniqueness fails in $H^{s}(\mathbb{R})$ for $s<-1 / 2$. Thus we have that the IVP for the dispersive models, like the $\mathrm{KdV}((1.8)$ with $k=1)$ and those in (1.1) with $N_{1}$ and $N_{3}$ in (1.7) exhibit "better" local existence properties than that of the parabolic equation in (1.9). 
Another interesting set of results are those concerned with the 3-D nonlinear wave equation

$$
\left\{\begin{array}{l}
\partial_{t}^{2} \omega-\Delta \omega=G\left(\nabla \omega, \partial_{t} \omega\right), \quad x \in \mathbb{R}^{3}, t \in \mathbb{R}, \\
\omega(x, 0)=f(x) \in H^{s}\left(\mathbb{R}^{3}\right), \\
\partial_{t} \omega(x, 0)=g(x) \in H^{s-1}\left(\mathbb{R}^{3}\right) .
\end{array}\right.
$$

Written as a quasi-linear hyperbolic system we have that (1.10) is locally wellposed for $s>\frac{n}{2}+1=5 / 2$ (see [K1]). On the other hand, the scaling argument suggests that for $G(\cdot)$ satisfying

$$
G\left(\lambda \nabla \omega, \lambda \partial_{t} \omega\right)=\lambda^{j} G\left(\nabla \omega, \partial_{t} \omega\right), \quad j=2, \ldots,
$$

one should have local well-posedness for

$$
s>s(j)=(5 j-7) /(2 j-2) .
$$

In [PS] G. Ponce and T. Sideris showed that this is the case if $j \geq 3$, and this is optimal, and that if $j=2$, then $s>2$ suffices for the local well-posedness of (1.10). H. Lindblad [L] gave examples of $G(\cdot)$ satisfying (1.11), with $j=2$, for which the corresponding IVP (1.10) is ill-posed for $s<2$. In [KM2] S. Klainerman and M. Machedon, improving their earlier result in [KM1] (which motivated those in [PS], [L]), showed that, for a special form of the quadratic nonlinearity in $\left(\nabla \omega, \partial_{t} \omega\right)$ the value suggested by the scaling in (1.12) can be reached. More precisely, for nonlinearities satisfying the so-called "null condition," i.e. $G\left(\omega, \nabla \omega, \partial_{t} \omega\right)=\phi(\omega)\left(\left(\partial_{t} \omega\right)^{2}-(\nabla \omega)^{2}\right)$, the IVP (1.10) is locally well-posed for $s>3 / 2$, the value suggested by the scaling argument, $(j=2$ in (1.12)).

Thus, similar to the results obtained by S. Klainerman and M. Machedon in [KM2], Theorems 1.5-1.7 suggest that the local existence theory for IVP (1.1) may depend on the structure of the nonlinear terms as well as its degree.

Our method of proof combines the ideas of J. Bourgain in [B] and those in [KPV3]. First we have the two parameter family spaces $X_{s, b}$ introduced in [B].

Definition. For $s, b \in \mathbb{R}, X_{s, b}$ denotes the completion of the Schwartz class $S\left(\mathbb{R}^{2}\right)$ with respect to the norm.

$$
\|F\|_{X_{s, b}}=\left(\int_{-\infty}^{\infty} \int_{-\infty}^{\infty}\left(1+\left|\tau-\xi^{2}\right|\right)^{2 b}(1+|\xi|)^{2 s}|\hat{F}(\xi, \tau)|^{2} d \xi d \tau\right)^{1 / 2}
$$

For $F \in X_{s, b}$ consider the bilinear operators

$$
\begin{gathered}
B_{1}(F, F)(x, t)=F^{2}(x, t), \\
B_{2}(F, F)(x, t)=(F \bar{F})(x, t),
\end{gathered}
$$

and

$$
B_{3}(F, F)(x, t)=\bar{F}^{2}(x, t),
$$

associated to the nonlinearities $N_{1}(\cdot), N_{2}(\cdot)$ and $N_{3}(\cdot)$ in (1.7) respectively.

Our well-posedness results for the IVP (1.1) are consequences of the following estimates for the bilinear forms (1.14)-(1.16).

Theorem 1.1. Given $s \in(-3 / 4,0]$ there exists $b \in(1 / 2,1)$ such that

$$
\left\|B_{1}(F, F)\right\|_{X_{s, b-1}} \leq c\|F\|_{X_{s, b}}^{2} .
$$


Theorem 1.2. Given $s \in(-1 / 4,0]$ there exists $b \in(1 / 2,1)$ such that

$$
\left\|B_{2}(F, F)\right\|_{X_{s, b-1}} \leq c\|F\|_{X_{s, b}}^{2} .
$$

Theorem 1.3. Given $s \in(-3 / 4,0]$ there exists $b \in(1 / 2,1)$ such that

$$
\left\|B_{3}(F, F)\right\|_{X_{s, b-1}} \leq c\|F\|_{X_{s, b}}^{2} .
$$

As in [KPV3] our proof of Theorems 1.1-1.3 uses elementary techniques somewhat similar to those found in the works of C. Fefferman and E. M. Stein [F1] on the $L^{4 / 3}\left(\mathbb{R}^{2}\right)$ restriction theorem for the Fourier transform to the circle and those of C. Fefferman [F2] for the $L^{4}\left(\mathbb{R}^{2}\right)$ boundedness of the Bochner-Riesz operator.

The following theorem shows that the results in Theorems 1.1-1.3 are sharp, except for the limiting cases which remain open.

\section{Theorem 1.4.}

i) For any $s<-3 / 4$ and any $b \in \mathbb{R}$ the estimate (1.17) fails.

ii) For any $s<-1 / 4$ and any $b \in \mathbb{R}$ the estimate (1.18) fails.

iii) For any $s<-3 / 4$ and any $b \in \mathbb{R}$ the estimate (1.19) fails.

Once the bilinear estimates (1.17)-(1.19) have been established we follow an approach similar to that given in [KPV2], [KPV3] to obtain the following local well-posedness results for the IVP (1.1) with nonlinear terms in (1.7).

Theorem 1.5. Let $s \in(-3 / 4,0]$. Then there exists $b \in(1 / 2,1)$ such that for any $u_{0} \in H^{s}(\mathbb{R})$ there exists $T=T\left(\left\|u_{0}\right\|_{H^{s}}\right)>0$ (with $T(\rho) \rightarrow \infty$ as $\rho \rightarrow 0$ ) and a unique solution $u(t)$ of the IVP (1.1), with the nonlinear term $N=N_{1}$ given by (1.7), satisfying

$$
u \in C\left([-T, T]: H^{s}(\mathbb{R})\right),
$$

and

$$
u^{2} \in X_{s, b-1}, \quad \partial_{t} u, \partial_{x}^{2} u \in X_{s-2, b-1} .
$$

Moreover, given $T^{\prime} \in(0, T)$ there exists $R=R\left(T^{\prime}\right)>0$ such that the map $\tilde{u}_{0} \rightarrow \tilde{u}(t)$ from $\left\{\tilde{u}_{0} \in H^{s}(\mathbb{R}):\left\|\tilde{u}_{0}-u_{0}\right\|_{H^{s}}<R\right\}$ into the class (1.20)-(1.21) with $T^{\prime}$ instead of $T$ is Lipschitz.

If in addition, $u_{0} \in H^{s^{\prime}}(\mathbb{R})$ with $s^{\prime}>s$, then the above results hold with $s^{\prime}$ instead of $s$ in the same time interval $[-T, T]$.

Theorem 1.6. For the IVP (1.1) with nonlinear term $N=N_{2}$ given in (1.7) the results in Theorem 1.4 (with un in (1.22) instead of uu) hold for $s \in(-1 / 4,0]$.

Theorem 1.7. For the IVP (1.1) with nonlinear term $N=N_{3}$ given in (1.7) the results in Theorem 1.4 (with $\bar{u} \bar{u}$ in (1.22) instead of uu) apply in the same interval $s \in(-3 / 4,0]$.

Next we consider the pbvp for the Schrödinger equation

$$
\left\{\begin{array}{l}
\partial_{t} u=i \partial_{x}^{2} u+N(u, \bar{u}), \quad x \in \mathbb{T}, t \in \mathbb{R}, \\
u(x, 0)=u_{0}(x)
\end{array}\right.
$$

with the quadratic homogeneous nonlinearities in (1.7). 
In [B] J. Bourgain showed that the IVP (1.23) with nonlinearity

$$
N\left(z_{1}, z_{2}\right)=\sum_{|\alpha| \leq 3} a_{\alpha} z_{1}^{\alpha_{1}} z_{2}^{\alpha_{2}}
$$

is locally well-posed in $L^{2}(\mathbb{T})$.

In the case of quadratic nonlinearities, i.e. $|\alpha| \leq 2$ in (1.24), this result follows as a consequence of the estimate due to A. Zygmund [Z],

$$
\left\|\sum_{n=-\infty}^{\infty} a_{n} e^{i\left(n x+n^{2} t\right)}\right\|_{L_{x, t}^{4}\left(\mathbb{T}^{2}\right)} \leq c\left(\sum_{n=-\infty}^{\infty}\left|a_{n}\right|^{2}\right)^{1 / 2}
$$

(observe that in $\mathbb{R}(1.25)$ corresponds to the case $p=r=6$ in (1.3)).

To state our result for the pbvp (1.23) we need the function spaces $Y_{s, b}$.

Definition. Let $\mathcal{Y}$ be the space of functions $F(\cdot)$ such that

(i) $F: \mathbb{T} \times \mathbb{R} \rightarrow \mathbb{C}$.

(ii) $F(x, \cdot) \in S(\mathbb{R})$ for each $x \in \mathbb{T}$.

(iii) $F(\cdot, t) \in C^{\infty}(\mathbb{T})$ for each $t \in \mathbb{R}$.

For $s, b \in \mathbb{R}, Y_{s, b}$ denotes the completion of $\mathcal{Y}$ with respect to the norm

$$
\|F\|_{Y_{s, b}}=\left(\sum_{n=-\infty}^{\infty} \int_{-\infty}^{\infty}\left(1+\left|\tau-n^{2}\right|\right)^{2 b}(1+|n|)^{2 s}|\hat{F}(n, \tau)|^{2} d \tau\right)^{1 / 2} .
$$

As in the case of the IVP (1.1), our well-posedness results for the pbvp (1.23) will be a consequence of the following estimates for the bilinear forms (1.14)-(1.16).

Theorem 1.8. Given $s \in(-1 / 2,0]$ there exists $b \in(1 / 2,1)$ such that

$$
\left\|B_{1}(F, F)\right\|_{Y_{s, b-1}} \leq c\|F\|_{Y_{s, b}}^{2} .
$$

Theorem 1.9. Given $s \in(-1 / 2,0]$ there exists $b \in(1 / 2,1)$ such that

$$
\left\|B_{3}(F, F)\right\|_{Y_{s, b-1}} \leq c\|F\|_{Y_{s, b}}^{2} .
$$

The following result shows the sharpness of (1.27)-(1.28).

\section{Theorem 1.10.}

(i) For any $s<-1 / 2$ and any $b \in \mathbb{R}$ the estimate (1.27) fails.

(ii) For any $s<-1 / 2$ and any $b \in \mathbb{R}$ the estimate (1.28) fails.

(iii) For any $s<0$ and any $b \in \mathbb{R}$ the estimate

$$
\left\|B_{2}(F, F)\right\|_{Y_{s, b-1}} \leq c\|F\|_{Y_{s, b}}^{2}
$$

fails.

Theorem 1.11. Let $s \in(-1 / 2,0]$. Then there exists $b \in(1 / 2,1)$ such that for any $u_{0} \in H^{s}(\mathbb{T})$ there exists $T=T\left(\left\|u_{0}\right\|_{H^{s}}\right)>0$ and a unique solution $u(t)$ of the pbvp (1.23), with the nonlinear term $N=N_{1}$ given in (1.7), satisfying

$$
u \in C\left([-T, T]: H^{s}(\mathbb{T})\right),
$$

and

$$
u \in Y_{s, b}
$$

$$
u^{2} \in Y_{s, b-1}, \quad \partial_{t} u, \partial_{x}^{2} u \in Y_{s-2, b-1} .
$$


Moreover, given $T^{\prime} \in(0, T)$ there exists $R=R\left(T^{\prime}\right)>0$ such that the map $\tilde{u}_{0} \rightarrow \tilde{u}(t)$ from $\left\{\tilde{u}_{0} \in H^{s}(\mathbb{T}):\left\|\tilde{u}_{0}-u_{0}\right\|_{H^{s}}<R\right\}$ into the class (1.30)-(1.31).

Theorem 1.12. for the pbvp (1.23) with nonlinearity $N=N_{3}$ given in (1.7) the results in Theorem 1.12 (with $\bar{u} \bar{u}$ in (1.32) instead of uu) apply in the same interval $s \in(-1 / 2,0]$.

We observe a loss of $1 / 4$ derivatives in each result corresponding to the pbvp (1.23) in comparison to the corresponding one for the IVP (1.1). In particular, for the nonlinearity $N_{2}(u, \bar{u})=c u \bar{u}$ we do not improve the $L^{2}$-result which follows from (1.25) (see $[\mathrm{B}]$ ).

This paper is organized as follows. Section 2 is concerned with the results for the IVP (1.1) with $N=N_{1}$, (i.e., Theorems 1.1, 1.4(i) and 1.5). Section 3 contains the proof of Theorems 1.2 and 1.4(ii) and section 4 the proof of Theorems 1.3 and 1.4(iii). Finally, in section 5 we include our result for the pvbp (1.23), Theorems 1.81.10. We remark that once that the bilinear estimates (1.27)-(1.28) (resp. (1.18)(1.19)) have been established, the proof of Theorems 1.11-1.12 (resp. Theorems 1.6-1.7) follows an argument similar to that provided in the proof of Theorem 1.5 in section 2 (see [KPV2], [KPV3]); therefore their proof will be omitted.

\section{Proof of Theorems 1.1, 1.4(i) And 1.5}

First we state some elementary calculus inequalities which are the main tools in the proof of Theorems 1.1-1.3.

Lemma 2.1. If $r>1 / 2$, then there exists $c>0$ such that

$$
\begin{gathered}
\int_{-\infty}^{\infty} \frac{d x}{(1+|x-\alpha|)^{2 r}(1+|x-\beta|)^{2 r}} \leq \frac{c}{(1+|\alpha-\beta|)^{2 r}}, \\
\int_{-\infty}^{\infty} \frac{d x}{(1+|x|)^{2 r}|\sqrt{\alpha-x}|} \leq \frac{c}{(1+|\alpha|)^{1 / 2}}, \\
\int_{-\infty}^{\infty} \frac{d x}{(1+|x-\alpha|)^{2(1-r)}(1+|x-\beta|)^{2 r}} \leq \frac{c}{(1+|\alpha-\beta|)^{2(1-r)}},
\end{gathered}
$$

and

$$
\int_{|x| \leq \beta} \frac{d x}{(1+|x|)^{2(1-r)}|\sqrt{\alpha-x}|} \leq c \frac{(1+\beta)^{2(r-1 / 2)}}{(1+|\alpha|)^{1 / 2}} .
$$

Next we define

$$
\rho=-s \in[0,3 / 4)
$$

and for $F \in X_{s, b}=X_{-\rho, b}$

$$
f(\xi, \tau) \equiv\left(1+\left|\tau-\xi^{2}\right|\right)^{b}(1+|\xi|)^{-\rho} \hat{F}(\xi, \tau) \in L^{2}\left(\mathbb{R}^{2}\right) .
$$

Thus

$$
\|f\|_{L_{\xi}^{2} L_{\tau}^{2}}=\|F\|_{X_{s, b}}
$$


and we can rewrite (1.17) in terms of $f$ as

$$
\begin{gathered}
\left\|B_{1}(F, F)\right\|_{X_{s, b-1}}=\left\|\left(1+\left|\tau-\xi^{2}\right|\right)^{b-1}(1+|\xi|)^{-\rho} \widehat{F^{2}}\right\|_{L_{\xi}^{2} L_{\tau}^{2}} \\
=\left\|\left(1+\left|\tau-\xi^{2}\right|\right)^{b-1}(1+|\xi|)^{-\rho}(\hat{F} * \hat{F})\right\|_{L_{\xi}^{2} L_{\tau}^{2}} \\
=c \| \frac{1}{\left(1+\left|\tau-\xi^{2}\right|\right)^{1-b}(1+|\xi|)^{\rho}} \\
\times \iint \frac{f\left(\xi_{1}, \tau_{1}\right)\left(1+\left|\xi_{1}\right|\right)^{\rho}}{\left(1+\left|\tau_{1}-\xi_{1}^{2}\right|\right)^{b}} \frac{f\left(\xi-\xi_{1}, \tau-\tau_{1}\right)\left(1+\left|\xi-\xi_{1}\right|\right)^{\rho}}{\left(1+\left|\tau-\tau_{1}-\left(\xi-\xi_{1}\right)^{2}\right|\right)^{b}} d \xi_{1} d \tau_{1} \|_{L_{\xi}^{2} L_{\tau}^{2}}
\end{gathered}
$$

Defining

$$
\begin{gathered}
\mathcal{B}_{1}(f, g, \rho, b)(\xi, \tau)=\frac{1}{\left(1+\left|\tau-\xi^{2}\right|\right)^{1-b}(1+|\xi|)^{\rho}} \\
\times \iint \frac{f\left(\xi_{1}, \tau_{1}\right)\left(1+\left|\xi_{1}\right|\right)^{\rho}}{\left(1+\left|\tau_{1}-\xi_{1}^{2}\right|\right)^{b}} \frac{g\left(\xi-\xi_{1}, \tau-\tau_{1}\right)\left(1+\left|\xi-\xi_{1}\right|\right)^{\rho}}{\left(1+\left|\tau-\tau_{1}-\left(\xi-\xi_{1}\right)^{2}\right|\right)^{b}} d \xi_{1} d \tau_{1},
\end{gathered}
$$

Theorem 1.1 can be restated as follows.

Theorem 2.2. Given $\rho=-s \in[0,3 / 4)$ there exists $b \in(1 / 2,1)$ such that

$$
\left\|\mathcal{B}_{1}(f, g, \rho, b)\right\|_{L_{\xi}^{2} L_{\tau}^{2}} \leq c\|f\|_{L_{\xi}^{2} L_{\tau}^{2}}\|g\|_{L_{\xi}^{2} L_{\tau}^{2}} .
$$

The proof of Theorem 2.2 will be based on the following three lemmas.

Lemma 2.3. If $b \in(1 / 2,1]$, then

$$
\left\|\mathcal{B}_{1}(f, g, 0, b)\right\|_{L_{\xi}^{2} L_{\tau}^{2}} \leq c\|f\|_{L_{\xi}^{2} L_{\tau}^{2}}\|g\|_{L_{\xi}^{2} L_{\tau}^{2}} .
$$

Proof. It suffices to see that for $b^{\prime}>1 / 2$ and $b \leq 1$

$$
\sup _{\xi, \tau} \frac{1}{\left(1+\left|\tau-\xi^{2}\right|\right)^{1-b}}
$$

$$
\times\left(\int_{-\infty}^{\infty} \int_{-\infty}^{\infty} \frac{d \tau_{1} d \xi_{1}}{\left(1+\left|\tau_{1}-\xi_{1}^{2}\right|\right)^{2 b^{\prime}}\left(1+\left|\tau-\tau_{1}-\left(\xi-\xi_{1}\right)^{2}\right|\right)^{2 b^{\prime}}}\right)^{1 / 2}<\infty .
$$

This stronger statement will be useful later on.

From (2.1) it follows that

$$
\begin{gathered}
\int_{-\infty}^{\infty} \frac{d \tau_{1}}{\left(1+\left|\tau_{1}-\xi_{1}^{2}\right|\right)^{2 b^{\prime}}\left(1+\left|\tau-\tau_{1}-\left(\xi-\xi_{1}\right)^{2}\right|\right)^{2 b^{\prime}}} \\
\leq \frac{c}{\left(1+\left|\tau-\xi^{2}+2 \xi_{1}\left(\xi-\xi_{1}\right)\right|\right)^{2 b^{\prime}}}
\end{gathered}
$$

To integrate in $\xi_{1}$ we change variables

$$
\eta=\tau-\xi^{2}+2 \xi_{1}\left(\xi-\xi_{1}\right)
$$

thus

$$
d \eta=2\left(\xi-2 \xi_{1}\right) d \xi_{1}
$$


and

$$
\xi_{1}=\frac{1}{2}\left(\xi \pm \sqrt{2 \tau-\xi^{2}-2 \eta}\right), \quad \text { i.e. }\left|2 \xi_{1}-\xi\right|=\left|\sqrt{2 \tau-\xi^{2}-2 \eta}\right|
$$

Hence

$$
d \xi_{1}=c \frac{d \eta}{\left|\sqrt{\tau-\eta-\xi^{2} / 2}\right|}
$$

and from (2.2) we get

$$
\begin{aligned}
\int_{-\infty}^{\infty} \frac{d \xi_{1}}{\left(1+\left|\tau-\xi^{2}+2 \xi_{1}\left(\xi-\xi_{1}\right)\right|\right)^{2 b^{\prime}}} & =c \int_{-\infty}^{\infty} \frac{d \eta}{(1+|\eta|)^{2 b^{\prime}}\left|\sqrt{\tau-\eta-\xi^{2} / 2}\right|} \\
& \leq \frac{c}{\left(1+\left|\tau-\xi^{2} / 2\right|\right)^{1 / 2}} .
\end{aligned}
$$

Thus the expression in (2.12) can be bounded by

$$
c \sup _{\xi, \tau} \frac{1}{\left(1+\left|\tau-\xi^{2}\right|\right)^{1-b}} \frac{1}{\left(1+\left|\tau-\xi^{2} / 2\right|\right)^{1 / 4}},
$$

which yields the result.

In the proof of the following two lemmas we will use the algebraic inequalities

$$
\left|\tau-\xi^{2}\right|+\left|\tau-\tau_{1}-\left(\xi-\xi_{1}\right)^{2}\right|+\left|\tau_{1}-\xi_{1}^{2}\right| \geq 2\left|\xi_{1}\left(\xi-\xi_{1}\right)\right|,
$$

and consequently

$$
\max \left\{\left|\tau-\xi^{2}\right| ;\left|\tau-\tau_{1}-\left(\xi-\xi_{1}\right)^{2}\right| ;\left|\tau_{1}-\xi_{1}^{2}\right|\right\} \geq \frac{2}{3}\left|\xi_{1}\left(\xi-\xi_{1}\right)\right| .
$$

Lemma 2.4. If $\rho=-s \in(1 / 2,3 / 4), b^{\prime}>1 / 2$ and $b \leq 5 / 4-\rho$, then

$$
\sup _{\xi, \tau} \frac{1}{\left(1+\left|\tau-\xi^{2}\right|\right)^{1-b}} \frac{1}{(1+|\xi|)^{\rho}}
$$

$$
\times\left(\iint_{A} \frac{\left|\xi_{1}\left(\xi-\xi_{1}\right)\right|^{2 \rho}}{\left(1+\left|\tau_{1}-\xi_{1}^{2}\right|\right)^{2 b^{\prime}}\left(1+\left|\tau-\tau_{1}-\left(\xi-\xi_{1}\right)^{2}\right|\right)^{2 b^{\prime}}} d \xi_{1} d \tau_{1}\right)^{1 / 2}<\infty,
$$

where $A=A(\xi, \tau)$ is defined as

$$
A=\left\{\left(\xi_{1}, \tau_{1}\right) \in \mathbb{R}^{2}:\left|\tau-\tau_{1}-\left(\xi-\xi_{1}\right)^{2}\right| \leq\left|\tau_{1}-\xi_{1}^{2}\right| \leq\left|\tau-\xi^{2}\right|\right\} .
$$

Proof. From (2.21) it follows that in $A$, if $\left|\xi_{1}\right| \geq 1$ then

$$
\left|\xi_{1}\left(\xi-\xi_{1}\right)\right| \leq 3\left|\tau-\xi^{2}\right| / 2
$$

and

$$
\left|\tau-\xi^{2}+2 \xi_{1}\left(\xi-\xi_{1}\right)\right| \leq\left|\tau_{1}-\xi_{1}^{2}\right|+\left|\tau-\tau_{1}-\left(\xi-\xi_{1}\right)^{2}\right| \leq 2\left|\tau-\xi^{2}\right| .
$$

Thus, from (2.1)

$$
\begin{gathered}
\int \frac{d \tau_{1}}{\left(1+\left|\tau_{1}-\xi_{1}^{2}\right|\right)^{2 b^{\prime}}\left(1+\left|\tau-\tau_{1}-\left(\xi-\xi_{1}\right)^{2}\right|\right)^{2 b^{\prime}}} \\
\leq c \frac{\psi\left(\left(\tau-\xi^{2}+2 \xi_{1}\left(\xi-\xi_{1}\right)\right) / 2\left(\tau-\xi^{2}\right)\right)}{\left(1+\left|\tau-\xi^{2}+2 \xi_{1}\left(\xi-\xi_{1}\right)\right|\right)^{2 b^{\prime}}}
\end{gathered}
$$


where $\psi \in C_{0}^{\infty}(\mathbb{R}), \psi \equiv 1$ in $[-1,1]$ and supp $\psi \subseteq(-2,2)$.

Combining the change of variables $(2.14)-(2.17)$ and (2.2) we obtain that

$$
\begin{aligned}
& \int \frac{\psi\left(\left(\tau-\xi^{2}+2 \xi_{1}\left(\xi-\xi_{1}\right)\right) / 2\left(\tau-\xi^{2}\right)\right)}{\left(1+\left|\tau-\xi^{2}+2 \xi_{1}\left(\xi-\xi_{1}\right)\right|\right)^{2 b^{\prime}}} d \xi_{1} \\
\leq & \int_{|\eta| \leq\left|\tau-\xi^{2}\right|} \frac{d \eta}{(1+|\eta|)^{2 b^{\prime}}\left|\sqrt{\tau-\eta-\xi^{2} / 2}\right|} \leq \frac{c}{\left(1+\left|\tau-\xi^{2} / 2\right|\right)^{1 / 2}} .
\end{aligned}
$$

Thus collecting the information in (2.24)-(2.27) the expression in (2.22) can be bounded by

$$
\sup _{\xi, \tau} \frac{1}{(1+|\xi|)^{\rho}} \frac{\left(1+\left|\tau-\xi^{2}\right|\right)^{\rho+b-1}}{\left(1+\left|\tau-\xi^{2} / 2\right|\right)^{1 / 4}}
$$

which yields the desired result.

Lemma 2.5. If $\rho=-s \in(1 / 2,3 / 4), b \in(1 / 2,1)$ and $b^{\prime} \geq \rho / 2-3 / 4+b$, then

$$
\sup _{\left|\xi_{1}\right| \geq 1} \sup _{\tau_{1}} \frac{1}{\left(1+\left|\tau_{1}-\xi_{1}^{2}\right|\right)^{b^{\prime}}}
$$

$\times\left(\iint_{\tilde{A}} \frac{\left|\xi_{1}\left(\xi-\xi_{1}\right)\right|^{2 \rho}}{(1+|\xi|)^{2 \rho}\left(1+\left|\tau-\xi^{2}\right|\right)^{2(1-b)}\left(1+\left|\tau-\tau_{1}-\left(\xi-\xi_{1}\right)^{2}\right|\right)^{2 b^{\prime}}} d \xi d \tau\right)^{1 / 2}<\infty$ with $\tilde{A}=\tilde{A}\left(\xi_{1}, \tau_{1}\right)$ defined as

$$
\tilde{A}=\left\{\begin{array}{r}
(\xi, \tau) \in \mathbb{R}^{2}:\left|\tau-\tau_{1}-\left(\xi-\xi_{1}\right)^{2}\right| \leq\left|\tau_{1}-\xi_{1}^{2}\right| \\
\text { and } \quad\left|\tau-\xi^{2}\right| \leq\left|\tau_{1}-\xi_{1}^{2}\right|
\end{array}\right\}
$$

Proof. From (2.21) it follows that in $\tilde{A}$

$$
\left|\xi_{1}\left(\xi-\xi_{1}\right)\right| \leq 3\left|\tau_{1}-\xi_{1}^{2}\right| / 2
$$

and

$$
\left|\tau_{1}-\xi_{1}^{2}+2 \xi_{1}\left(\xi-\xi_{1}\right)\right| \leq\left|\tau-\xi^{2}\right|+\left|\tau-\tau_{1}-\left(\xi-\xi_{1}\right)^{2}\right| \leq 2\left|\tau_{1}-\xi_{1}^{2}\right| .
$$

Thus, as in (2.3) we obtain

$$
\begin{gathered}
\int \frac{d \tau}{\left(1+\left|\tau-\xi^{2}\right|\right)^{2(1-b)}\left(1+\left|\tau-\tau_{1}-\left(\xi-\xi_{1}\right)^{2}\right|\right)^{2 b^{\prime}}} \\
\quad \leq c \frac{\psi\left(\left(\tau-\xi_{1}^{2}+2 \xi_{1}\left(\xi-\xi_{1}\right)\right) / 2\left(\tau_{1}-\xi_{1}\right)\right)}{\left(1+\left|\tau_{1}-\xi_{1}^{2}+2 \xi_{1}\left(\xi_{1}-\xi\right)\right|\right)^{2(1-b)}} .
\end{gathered}
$$

Define, for a set $C \subseteq \mathbb{R}^{2}$

$$
I(C)=\frac{1}{\left(1+\left|\tau_{1}-\xi_{1}^{2}\right|\right)^{b^{\prime}}}\left(\int_{C} \frac{\left|\xi_{1}\left(\xi-\xi_{1}\right)\right|^{2 \rho} d \xi}{(1+|\xi|)^{2 \rho}\left(1+\left|\tau_{1}-\xi_{1}^{2}+2 \xi_{1}\left(\xi_{1}-\xi\right)\right|\right)^{2(1-b)}}\right)^{1 / 2} .
$$

Our aim is to bound $I(D)$, where

$$
D=D\left(\xi_{1}, \tau_{1}\right)=\left\{\xi \in \mathbb{R}:\left|\tau_{1}-\xi_{1}^{2}+2 \xi_{1}\left(\xi_{1}-\xi\right)\right| \leq 2\left|\tau_{1}-\xi_{1}^{2}\right|\right\},
$$


uniformly in $\left(\xi_{1}, \tau_{1}\right) \in \mathbb{R}^{2}$. We divide $D$ into two subdomains $D_{1}$ and $D_{2}$. In

$$
D_{1}=\left\{\xi \in D:\left|2 \xi_{1}\left(\xi_{1}-\xi\right)\right| \leq\left|\tau_{1}-\xi_{1}^{2}\right| / 2\right\}
$$

one has that

$$
\left|\tau_{1}-\xi_{1}^{2}\right| \leq 2\left|\tau_{1}-\xi_{1}^{2}+2 \xi_{1}\left(\xi_{1}-\xi\right)\right|
$$

and consequently

$$
I\left(D_{1}\right) \leq \frac{\left(1+\left|\tau_{1}-\xi_{1}^{2}\right|\right)^{\rho}}{\left(1+\left|\tau_{1}-\xi_{1}^{2}\right|\right)^{1-b+b^{\prime}}}\left(\int_{D_{1}} \frac{d \xi}{(1+|\xi|)^{2 \rho}}\right)^{1 / 2}<c .
$$

We subdivide $D_{2}$, i.e.

$$
D_{2}=\left\{\xi \in D:\left|\tau_{1}-\xi_{1}^{2}\right| / 4 \leq\left|\xi_{1}\left(\xi_{1}-\xi\right)\right| \leq 3\left|\tau_{1}-\xi_{1}^{2}\right| / 2\right\},
$$

into three pieces. First

$$
D_{2,1}=\left\{\xi \in D_{2}:|\xi| / 4 \leq\left|\xi_{1}\right| \leq 100|\xi|\right\} .
$$

In this set

$$
|\xi|^{2} \sim\left|\xi_{1}\right|^{2} \geq c\left(1+\left|\tau_{1}-\xi_{1}^{2}\right|\right)
$$

Therefore using the change of variables

$$
\eta=\tau_{1}-\xi_{1}^{2}+2 \xi_{1}\left(\xi_{1}-\xi\right), \quad d \eta=-2 \xi_{1} d \xi,
$$

we obtain

$$
\begin{aligned}
I\left(D_{2,1}\right) & \leq c\left(1+\left|\tau_{1}-\xi_{1}^{2}\right|\right)^{\rho / 2-b^{\prime}}\left(\int_{|\eta| \leq 2\left|\tau_{1}-\xi_{1}^{2}\right|} \frac{1}{\left|\xi_{1}\right|(1+|\eta|)^{2(1-b)}} d \eta\right)^{1 / 2} \\
& \leq c\left(1+\left|\tau_{1}-\xi_{1}^{2}\right|\right)^{\rho / 2-b^{\prime}+3 / 4+b} \leq c .
\end{aligned}
$$

In

$$
D_{2,2}=\left\{\xi \in D_{2}: 1 \leq\left|\xi_{1}\right| \leq|\xi| / 4\right\}
$$

using (2.21) one has

$$
\begin{aligned}
I\left(D_{2,2}\right) & \leq c\left(1+\left|\tau_{1}-\xi_{1}^{2}\right|\right)^{-b^{\prime}}\left(\int_{|\eta| \leq 2\left|\tau_{1}-\xi_{1}^{2}\right|} \frac{\left|\xi_{1}\right|^{2 \rho}}{\left|\xi_{1}\right|(1+|\eta|)^{2(1-b)}} d \eta\right)^{1 / 2} \\
& \leq c\left(1+\left|\tau_{1}-\xi_{1}^{2}\right|\right)^{2 \rho-1-b^{\prime}+\rho-1 / 2+b-1 / 2} .
\end{aligned}
$$

Finally we consider

$$
D_{2,3}=\left\{\xi \in D_{2}: 100|\xi| \leq\left|\xi_{1}\right|\right\} .
$$

In this region

$$
\left|\xi_{1}\right|^{2} \sim\left|\tau_{1}-\xi_{1}^{2}\right|
$$

Hence,

$$
I\left(D_{2,3}\right) \leq c\left(1+\left|\tau_{1}-\xi_{1}^{2}\right|\right)^{\rho-b^{\prime}-3 / 4+b} \leq c,
$$

which completes the proof of (2.29). 
Proof of Theorem 2.2. First we consider the case $s=0$. Combining CauchySchwarz inequality, (2.12) and Fubini's theorem, it follows that

$$
\begin{aligned}
& \left\|\frac{1}{\left(1+\left|\tau-\xi^{2}\right|\right)^{1-b}} \iint \frac{f\left(\xi_{1}, \tau_{1}\right)}{\left(1+\left|\tau_{1}-\xi_{1}^{2}\right|\right)^{b^{\prime}}} \frac{g\left(\xi-\xi_{1}, \tau-\tau_{1}\right)}{\left(1+\left|\tau-\tau_{1}-\left(\xi-\xi_{1}\right)^{2}\right|\right)^{b^{\prime}}} d \xi_{1} d \tau_{1}\right\|_{L^{2} L^{2}} \\
\leq & \left\|\frac{1}{\left(1+\left|\tau-\xi^{2}\right|\right)^{1-b}}\left(\iint \frac{d \xi_{1} d \tau_{1}}{\left(1+\left|\tau-\tau_{1}-\left(\xi-\xi_{1}\right)^{2}\right|\right)^{2 b^{\prime}}}\right)^{1 / 2}\right\|_{L_{\xi}^{\infty} L_{\tau}^{\infty}} \\
\times & \left\|\left(\iint\left|f\left(\xi_{1}, \tau_{1}\right)\right|^{2}\left|g\left(\xi-\xi_{1}, \tau-\tau_{1}\right)\right|^{2} d \xi_{1} d \tau_{1}\right)^{1 / 2}\right\|_{L_{\xi}^{2} L_{\tau}^{2}} \\
\leq & c\|f\|_{L_{\xi}^{2} L_{\tau}^{2}}\|g\|_{L_{\xi}^{2} L_{\tau}^{2}}
\end{aligned}
$$

for any $b^{\prime}>1 / 2$ and $b \leq 1$. Taking $b=b^{\prime}$ we obtain the result.

Next we consider the case $\rho=-s \in(1 / 2,3 / 4)$. We observe that if

$$
\text { either }\left|\xi_{1}\right| \leq 1 \text { or }\left|\xi-\xi_{1}\right| \leq 1 \text {, }
$$

then

$$
\left(1+\left|\xi_{1}\right|\right)^{\rho}\left(1+\left|\xi-\xi_{1}\right|\right)^{\rho} \leq c(1+|\xi|)^{\rho}
$$

which reduces the estimate to the previous case $s=0$. Therefore, we assume

$$
\left|\xi_{1}\right| \geq 1 \text { and }\left|\xi-\xi_{1}\right| \geq 1 \text {. }
$$

Also by symmetry we can restrict ourselves to the case

$$
\left|\tau-\tau_{1}-\left(\xi-\xi_{1}\right)^{2}\right| \leq\left|\tau_{1}-\xi_{1}^{2}\right| \text {. }
$$

Now we split the domain of integration into two pieces

$$
\left|\tau_{1}-\xi_{1}^{2}\right| \leq\left|\tau-\xi^{2}\right| \quad \text { and } \quad\left|\tau-\xi^{2}\right| \leq\left|\tau_{1}-\xi_{1}^{2}\right| .
$$

In the first part, $\left(\left|\tau_{1}-\xi_{1}^{2}\right| \leq\left|\tau-\xi^{2}\right|\right)$ we combine Cauchy-Schwarz and (2.22) with $b=b^{\prime}$, as in (2.49), to obtain the result. In the second part we use duality, Cauchy-Schwarz and (2.29) with $b=b^{\prime}$ to complete the result.

Corollary 2.5. If $\rho=-s \in(1 / 2,3 / 4), b \in(1 / 2,5 / 4-\rho]$ and $b^{\prime}>1 / 2$ with $b-b^{\prime} \in(0,3 / 4-\rho / 2]$, then

$$
\left\|B_{1}(F, F)\right\|_{X_{s, b-1}} \leq c\|F\|_{X_{s, b^{\prime}}}^{2} .
$$

Moreover (2.55) holds for $s=0$, and $1 / 2<b<b^{\prime} \leq 1$.

Proof of Theorem 1.4(i). For $N \in \mathbb{Z}^{+}$define

$$
f_{N}(\xi, \tau)=\psi(\xi-N) \psi\left(\tau-\xi^{2}\right)
$$

and

$$
g_{N}(\xi, \tau)=\psi(\xi+N) \psi\left(\tau-\xi^{2}\right)
$$

where $\psi \in C^{\infty}(\mathbb{R}), \psi \geq 0, \psi \equiv 1$ in $[-1,1]$ and $\operatorname{supp} \psi \subseteq[-2,2]$. A simple computation shows that for $N$ large

$$
\left(f_{N} * g_{N}\right)(\xi, \tau) \geq \frac{c}{N} \psi\left(2 N^{2}+\tau / N\right) \psi(\xi) .
$$


Thus (2.10) implies that

$$
\frac{1}{N^{2(1-b)}} \frac{N^{2 \rho}}{N^{1 / 2}} \leq c
$$

Now, taking

$$
h_{N}(\xi, \tau)=\psi(\xi+N) \psi\left(\tau+\xi^{2}\right)
$$

we have that for $N$ large

$$
\left(f_{N} * h_{N}\right)(\xi, \tau) \cong c \chi_{R}(\xi, \tau)
$$

where $\chi_{A}(\cdot)$ denotes the characteristic function of the set $A$, and $R$ is the rectangle of dimensions $c N \times N^{-1}$ centered in the origin with longest side pointing in the $(1,2 N)$ direction.

Thus, (2.10) tells that

$$
\frac{1}{N^{1-b}} \frac{N^{2 \rho}}{N^{2 b}} \leq c
$$

Combining (2.59), (2.62) and letting $N$ tend to infinity, we obtain that

$$
\rho \leq \min \left\{\frac{5}{4}-b ; \frac{b}{2}+\frac{1}{2}\right\}
$$

which completes the proof of Theorem 1.4(i).

Next, we deduce general estimates which are needed in the proof of Theorems $1.5-1.7$.

We denote by $\left\{e^{i t \partial_{x}^{2}}\right\}_{t=-\infty}^{\infty}$ the unitary group describing the solution of the linear IVP associated to (1.1).

$$
\left\{\begin{array}{l}
\partial_{t} u-i \partial_{x}^{2} u=0, \\
u(x, 0)=u_{0}(x)
\end{array} \quad t, x \in \mathbb{R}\right.
$$

where

$$
u(x, t)=e^{i t \partial_{x}^{2}} u_{0}(x)=c\left(e^{-i t \xi^{2}} \hat{u}_{0}(\xi)\right)^{\vee}(x) .
$$

We shall use the notations

$$
\begin{gathered}
\|f\|_{L_{x}^{p} L_{t}^{q}=}\left(\int_{-\infty}^{\infty}\left(\int_{-\infty}^{\infty}|f(t, x)|^{q} d t\right)^{p / q} d x\right)^{1 / p}, \\
\widehat{J^{s} h}(\xi)=(1+|\xi|)^{s} \hat{h}(\xi)
\end{gathered}
$$

and

$$
\widehat{\Lambda^{b}} g(\tau)=(1+|\tau|)^{b} \hat{g}(\tau)
$$

The identity

$$
\|F\|_{X_{s, b}}=\left\|\Lambda^{b} J^{s} e^{i t \partial_{x}^{2}} F\right\|_{L_{x}^{2} L_{t}^{2}}
$$

describes the relationship between the spaces $X_{s, b}$ 's and the group $\left\{e^{i t \partial_{x}^{2}}\right\}_{t=-\infty}^{\infty}$.

Let $\psi \in C_{0}^{\infty}(\mathbb{R})$ with $\psi \equiv 1$ on $[-1,1]$ and $\operatorname{supp} \psi \subseteq(-2,2)$. 
Lemma 2.6. If $s \leq 0$ and $b \in(1 / 2,1]$, then for $\delta \in(0,1)$

$$
\begin{gathered}
\left\|\psi\left(\delta^{-1} t\right) e^{i t \partial_{x}^{2}} u_{0}\right\|_{X_{s, b}} \leq c \delta^{(1-2 b) / 2}\left\|u_{0}\right\|_{H^{s}}, \\
\left\|\psi\left(\delta^{-1} t\right) F\right\|_{X_{s, b}} \leq c \delta^{(1-2 b) / 2}\|F\|_{X_{s, b}}, \\
\left\|\psi\left(\delta^{-1} t\right) \int_{0}^{t} e^{i\left(t-t^{\prime}\right) \partial_{x}^{2}} F\left(t^{\prime}\right) d t^{\prime}\right\|_{X_{s, b}} \leq c \delta^{(1-2 b) / 2}\|F\|_{X_{s, b-1}}
\end{gathered}
$$

and

$$
\sup _{t}\left\|\psi\left(\delta^{-1} t\right) \int_{0}^{t} e^{i\left(t-t^{\prime}\right) \partial_{x}^{2}} F\left(t^{\prime}\right) d t^{\prime}\right\|_{H^{s}} \leq c \delta^{(1-2 b) / 2}\|F\|_{X_{s, b-1}} .
$$

Proof. The proof of (2.70)-(2.72) is similar to that in [KPV2] (Lemmas 3.1-3.3) (see also [KPV3]) for the linear group $\{W(t)\}_{-\infty}^{\infty}$ associated to the linearized KdV equation.

Proof of Theorem 1.5. Using a scaling argument it follows that if $u=u(x, t)$ is a solution of the IVP (1.1) then for any $\lambda>0$

$$
u_{\lambda}(x, t)=\lambda^{2} u\left(\lambda x, \lambda^{2} t\right)
$$

also solves the equation in (1.1) with initial data

$$
u_{\lambda}(x, 0)=\lambda^{2} u(\lambda x, 0)
$$

Thus for $s \leq 0$

$$
\left\|u_{\lambda}(\cdot, 0)\right\|_{H^{s}}=\mathcal{O}\left(\lambda^{3 / 2+s}\right) \quad \text { as } \quad \lambda \rightarrow 0 .
$$

Since we are considering $s \in(-3 / 4,0]$, we can restrict ourselves to solve the IVP (1.1) with data $u_{0} \in H^{s}(\mathbb{R})$ such that

$$
\left\|u_{0}\right\|_{H^{s}}=\mu<<1,
$$

and use (2.74) to extend the result for data in $H^{s}(\mathbb{R})$ of arbitrary size.

For $u_{0} \in H^{s}(\mathbb{R}), s \in(-3 / 4,0]$, satisfying (2.77) we define the operator

$$
\Lambda_{u_{0}}(\omega)=\Lambda(\omega)=\psi(t) e^{i t \partial_{x}^{2}} u_{0}+c_{1} \psi(t) \int_{0}^{t} e^{i\left(t-t^{\prime}\right) \partial_{x}^{2}} u^{2}\left(t^{\prime}\right) d t^{\prime} .
$$

Our goal is to show that $\Lambda(\cdot)$ defines a contraction map on

$$
D_{s, b}(2 c \mu)=\left\{\omega \in X_{s, b}:\|\omega\|_{X_{s, b}} \leq 2 c \mu\right\}
$$

for any $b \in(1 / 2,1)$.

Combining (2.70), (2.72) with $\delta=1$ and (1.17), we find that

$$
\begin{aligned}
\|\Lambda(\omega)\|_{X_{s, b}} & \leq c \mu+c\left\|\omega^{2}\right\|_{X_{s, b-1}} \\
& \leq c \mu+c\|\omega\|_{X_{s, b}}^{2} \\
& \leq c \mu+c(2 c \mu)^{2} \leq 2 c \mu
\end{aligned}
$$

if we choose $\mu$ in (2.77) such that

$$
4 c^{2} \mu \leq 1 / 2 .
$$


Similarly

$$
\begin{aligned}
\|\Lambda(\omega)-\Lambda(\widetilde{\omega})\|_{X_{s, b}} & \leq c\left\|\psi(t) \int_{0}^{t} e^{i\left(t-t^{\prime}\right) \partial_{x}^{2}}\left(\omega^{2}-\widetilde{\omega}^{2}\right) d t^{\prime}\right\|_{X_{s, b}} \\
& \leq c\left\|\omega-\widetilde{\omega}^{2}\right\|_{X_{s, b-1}}=c\|(\omega+\widetilde{\omega})(\omega-\widetilde{\omega})\|_{X_{s, b-1}} \\
& \leq 4 c^{2} \mu\|\omega-\widetilde{\omega}\|_{X_{s, b}} \leq \frac{1}{2}\|\omega-\widetilde{\omega}\|_{X_{s, b}} .
\end{aligned}
$$

Thus, $\Lambda(\cdot)$ defines a contraction map, and consequently there exists a unique $u \in$ $D_{s, b}(2 c \mu)$ such that

$$
u(t)=\psi(t)\left(e^{i t \partial_{x}^{2}} u_{0}+c_{1} \int_{0}^{t} e^{i\left(t-t^{\prime}\right) \partial_{x}^{2}} u^{2}\left(t^{\prime}\right) d t^{\prime}\right) .
$$

Therefore in the time interval $[-1,1]$ solves the integral equation associated to the IVP (1.1) with $N=N_{1}(\cdot)$ given by (1.7). Since $u \in X_{s, b}$, with $b>1 / 2$, by the Sobolev embedding theorem and (2.69) it follows that

$$
\left.u\right|_{[-1,1]} \in C\left([-1,1]: H^{s}(\mathbb{R})\right) .
$$

Finally we explain how to extend the uniqueness result in $D_{s, b}(2 c \mu)$ to the whole space $X_{s, b}$. First we restrict the time interval, i.e. consider $\Lambda_{u_{0}}(\cdot)$ in $(2.78)$ with $\psi\left(\delta^{-1} t\right), \delta \in(0,1)$, instead of $\psi(t)$. Arguing as in [KPV2] we obtain estimates similar to those in $(2.80),(2.82)$ but with a factor $\delta^{\theta_{0}}, \theta_{0}=\theta_{0}\left(b, b^{\prime}\right)>0$, in the left side, which allows us to establish the contraction principle in $D_{s, b}(R)$ with $R(\delta) \rightarrow \infty$ as $\delta \rightarrow 0$ (for details see [KPV2]).

\section{Proof of Theorems 1.2, 1.4(ii) And 1.6}

Define

$$
\rho=-s \in[0,1 / 4)
$$

and for $F \in X_{s, b}=X_{-\rho, b}$

$$
f(\xi, \tau)=\left(1+\left|\tau-\xi^{2}\right|\right)^{b}(1+|\xi|)^{-\rho} \hat{F}(\xi, \tau) \in L^{2}\left(\mathbb{R}^{2}\right),
$$

thus

$$
\|f\|_{L_{\xi}^{2} L_{\tau}^{2}}=\|F\|_{X_{s, b}}
$$

Since

$$
\overline{\hat{F}}(\xi, \tau)=\hat{\bar{F}}(-\xi,-\tau)
$$

it follows that

$$
\hat{\bar{F}}(\xi, \tau)=\frac{(1+|\xi|)^{\rho}}{\left(1+\left|\tau+\xi^{2}\right|\right)^{b}} \bar{f}(-\xi,-\tau)
$$

with

$$
\|f\|_{L_{\xi}^{2} L_{\tau}^{2}}=\|F\|_{X_{s, b}} .
$$


Thus we can rewrite (1.18) in terms of $f$ as

$$
\begin{aligned}
& \left\|B_{2}(F, F)\right\|_{X_{s, b-1}}=\left\|\left(1+\left|\tau-\xi^{2}\right|\right)^{b-1}(1+|\xi|)^{-\rho} \widehat{F \bar{F}}\right\|_{L_{\xi}^{2} L_{\tau}^{2}} \\
= & \left\|\left(1+\left|\tau-\xi^{2}\right|\right)^{b-1}(1+|\xi|)^{-\rho}(\hat{F} * \hat{\bar{F}})\right\|_{L_{\xi}^{2} L_{\tau}^{2}} \\
= & c \| \frac{1}{\left(1+\left|\tau-\xi^{2}\right|\right)^{1-b}(1+|\xi|)^{\rho}} \\
& \times \iint \frac{f\left(\xi-\xi_{1}, \tau-\tau_{1}\right)\left(1+\left|\xi-\xi_{1}\right|\right)^{\rho}}{\left(1+\left|\tau-\tau_{1}-\left(\xi-\xi_{1}\right)^{2}\right|\right)^{b}} \frac{\bar{f}\left(-\xi_{1},-\tau_{1}\right)\left(1+\left|\xi_{1}\right|\right)^{\rho}}{\left(1+\left|\tau_{1}+\xi_{1}^{2}\right|\right)^{b}} d \xi_{1} d \tau_{1} \|_{L_{\xi}^{2} L_{\tau}^{2}} .
\end{aligned}
$$

Defining

$$
\begin{gathered}
\mathcal{B}_{2}(f, g, \rho, b)(\xi, \tau)=\frac{1}{\left(1+\left|\tau-\xi^{2}\right|\right)^{1-b}(1+|\xi|)^{\rho}} \\
\times \iint \frac{f\left(\xi_{1}, \tau_{1}\right)\left(1+\left|\xi_{1}\right|\right)^{\rho}}{\left(1+\left|\tau_{1}+\xi_{1}^{2}\right|\right)^{b}} \frac{g\left(\xi-\xi_{1}, \tau-\tau_{1}\right)\left(1+\left|\xi-\xi_{1}\right|\right)^{\rho}}{\left(1+\left|\tau-\tau_{1}-\left(\xi-\xi_{1}\right)^{2}\right|\right)^{b}} d \xi_{1} d \tau_{1},
\end{gathered}
$$

Theorem 1.2 can be rewritten as follows.

Theorem 3.1. Given $\rho=-s \in[0,1 / 4)$ there exists $b \in(1 / 2,1)$ such that

$$
\left\|\mathcal{B}_{2}(f, g, \rho, b)\right\|_{L_{\xi}^{2} L_{\tau}^{2}} \leq c\|f\|_{L_{\xi}^{2} L_{\tau}^{2}}\|g\|_{L_{\xi}^{2} L_{\tau}^{2}} .
$$

The proof of Theorem 3.1 will be deduced from the following lemmas.

Lemma 3.2. If $b \in(1 / 2,1]$, then

$$
\left\|\mathcal{B}_{2}(f, g, 0, b)\right\|_{L_{\xi}^{2} L_{\tau}^{2}} \leq c\|f\|_{L_{\xi}^{2} L_{\tau}^{2}}\|g\|_{L_{\xi}^{2} L_{\tau}^{2}} .
$$

Proof. It will be shown that

$$
\begin{gathered}
\sup _{|\xi| \geq 1} \sup _{\tau} \frac{1}{\left(1+\left|\tau-\xi^{2}\right|\right)^{1-b}} \\
\times\left(\iint \frac{d \tau_{1} d \xi_{1}}{\left(1+\left|\tau_{1}+\xi_{1}^{2}\right|\right)^{2 b}\left(1+\left|\tau-\tau_{1}-\left(\xi-\xi_{1}\right)^{2}\right|\right)^{2 b}}\right)^{1 / 2}<\infty
\end{gathered}
$$

and

$$
\begin{gathered}
\sup _{\xi_{1}, \tau_{1}} \frac{1}{\left(1+\left|\tau_{1}+\xi_{1}^{2}\right|\right)^{b}} \\
\times\left(\int_{|\xi| \leq 1} \int \frac{d \tau d \xi}{\left(1+\left|\tau-\xi^{2}\right|\right)^{2(1-b)}\left(1+\left|\tau-\tau_{1}-\left(\xi-\xi_{1}\right)^{2}\right|\right)^{2 b}}\right)^{1 / 2}<\infty .
\end{gathered}
$$

It is easy to see that (3.10) follows by combining (3.11)-(3.12), Cauchy-Schwarz and duality.

To prove (3.11) we first use (2.1) to find that

$$
\int \frac{d \tau_{1}}{\left(1+\left|\tau_{1}+\xi_{1}^{2}\right|\right)^{2 b}\left(1+\left|\tau-\tau_{1}-\left(\xi-\xi_{1}\right)^{2}\right|\right)^{2 b}} \leq \frac{c}{\left(1+\left|\tau-\xi^{2}+2 \xi \xi_{1}\right|\right)^{2 b}} .
$$


Next, changing variables

$$
\eta_{1}=\tau-\xi^{2}+2 \xi \xi_{1}, \quad d \eta_{1}=2 \xi d \xi_{1},
$$

it follows that

$$
\int \frac{d \xi_{1}}{\left(1+\left|\tau-\xi^{2}+2 \xi \xi_{1}\right|\right)^{2 b}} \leq c \int \frac{d \eta_{1}}{|\xi|\left(1+\left|\eta_{1}\right|\right)^{2 b}} \leq \frac{c^{\prime}}{|\xi|}
$$

Inserting (3.15) in (3.13) we obtain (3.11). To prove (3.12) we use (2.3) to conclude that

$$
\begin{aligned}
& \int_{|\xi| \leq 1}\left(\int \frac{d \tau}{\left(1+\left|\tau-\xi^{2}\right|\right)^{2(1-b)}\left(1+\left|\tau-\tau_{1}-\left(\xi-\xi_{1}\right)^{2}\right|\right)^{2 b}}\right) d \xi \\
\leq & c \int_{|\xi| \leq 1} \frac{d \xi}{\left(1+\left|\tau_{1}+\xi_{1}^{2}-2 \xi \xi_{1}\right|\right)^{2(1-b)}} \leq 2 c,
\end{aligned}
$$

which yields (3.12).

In the next proofs we will use the following algebraic relations

$$
\left|\tau-\tau_{1}-\left(\xi-\xi_{1}\right)^{2}\right|+\left|\tau_{1}+\xi_{1}^{2}\right|+\left|\tau-\xi^{2}\right| \geq 2\left|\xi \xi_{1}\right|
$$

and consequently

$$
\max \left\{\left|\tau-\tau_{1}-\left(\xi-\xi_{1}\right)^{2}\right| ;\left|\tau_{1}+\xi_{1}^{2}\right| ;\left|\tau-\xi^{2}\right|\right\} \geq 2\left|\xi \xi_{1}\right| / 3 .
$$

Lemma 3.3. If $\rho=-s \in[0,1 / 4)$, then there exists $b>1 / 2$ such that

$$
\sup _{|\xi| \geq 1} \sup _{\tau} \frac{1}{\left(1+\left|\tau-\xi^{2}\right|\right)^{1-b}} \frac{1}{(1+|\xi|)^{\rho}}
$$

$$
\times\left(\iint_{A_{1}} \frac{\left|\xi_{1}\left(\xi-\xi_{1}\right)\right|^{2 \rho}}{\left(1+\left|\tau_{1}+\xi_{1}^{2}\right|\right)^{2 b}\left(1+\left|\tau-\tau_{1}-\left(\xi-\xi_{1}\right)^{2}\right|\right)^{2 b}} d \xi_{1} d \tau_{1}\right)^{1 / 2}<\infty
$$

where $A_{1}=A_{1}(\xi, \tau)$ is defined as

$$
A_{1}=\left\{\begin{array}{c}
\left(\xi_{1}, \tau_{1}\right) \in \mathbb{R}^{2}:\left|\xi_{1}\right| \geq 10, \quad\left|\xi-\xi_{1}\right| \geq 10 \\
\left|\tau-\tau_{1}-\left(\xi-\xi_{1}\right)^{2}\right| \leq\left|\tau-\xi^{2}\right| \\
\text { and }\left|\tau_{1}+\xi_{1}^{2}\right| \leq\left|\tau-\xi^{2}\right|
\end{array}\right\}
$$

Proof. From (2.1) it follows that

$$
\begin{aligned}
& \int_{\tau_{1} \in A_{1}} \frac{d \tau_{1}}{\left(1+\left|\tau_{1}+\xi_{1}^{2}\right|\right)^{2 b}\left(1+\left|\tau-\tau_{1}-\left(\xi-\xi_{1}\right)^{2}\right|\right)^{2 b}} \\
\leq & \frac{\psi\left(\left(\tau-\xi^{2}+2 \xi \xi_{1}\right) / 2\left(\tau-\xi^{2}\right)\right)}{\left(1+\left|\tau-\xi^{2}+2 \xi \xi_{1}\right|\right)^{2 b}},
\end{aligned}
$$

and changing variable as in (3.14)

$$
\int_{\left|\tau-\xi^{2}+2 \xi \xi_{1}\right| \leq 2\left|\tau-\xi^{2}\right|} \frac{d \xi_{1}}{\left(1+\left|\tau-\xi^{2}+2 \xi \xi_{1}\right|\right)^{2 b}} \leq c \frac{1}{|\xi|} \int_{\left|\eta_{1}\right| \leq 2\left|\tau-\xi^{2}\right|} \frac{d \eta_{1}}{\left(1+\left|\eta_{1}\right|\right)^{2 b}} \leq c
$$

since $|\xi| \geq 1$. 
In $A_{1},(3.18)$ and the hypothesis guarantee that there exists $b>1 / 2$ such that

$$
\left|\xi_{1}\left(\xi-\xi_{1}\right)\right|^{2 \rho} \leq c\left|\tau-\xi^{2}\right|^{4 \rho} \leq c\left|\tau-\xi^{2}\right|^{2(1-b)},
$$

thus by inserting (3.21)-(3.23) in (3.19) we obtain the desired result.

Lemma 3.4. If $\rho=-s \in[0,1 / 4)$, then there exists $b>1 / 2$ such that

$$
\begin{gathered}
\sup _{\left|\xi_{1}\right| \geq 10} \sup _{\tau_{1}} \frac{1}{\left(1+\left|\tau_{1}+\xi_{1}^{2}\right|\right)^{b}} \\
\times\left(\iint_{|\xi| \leq 1} \frac{\left|\xi_{1}\left(\xi-\xi_{1}\right)\right|^{2 \rho} d \xi d \tau}{(1+|\xi|)^{2 \rho}\left(1+\left|\tau-\tau_{1}-\left(\xi-\xi_{1}\right)^{2}\right|\right)^{2 b}\left(1+\left|\tau-\xi^{2}\right|\right)^{2(1-b)}}\right)^{1 / 2}<\infty .
\end{gathered}
$$

Proof. Using (2.3) we find that

$$
\int \frac{d \tau}{\left(1+\left|\tau-\tau_{1}-\left(\xi-\xi_{1}\right)^{2}\right|\right)^{2 b}\left(1+\left|\tau-\xi^{2}\right|\right)^{2(1-b)}} \leq \frac{c}{\left(1+\left|\tau_{1}+\xi_{1}^{2}-2 \xi \xi_{1}\right|\right)^{2(1-b)}},
$$

and by changing variables

$$
\eta=\tau_{1}+\xi_{1}^{2}-2 \xi \xi_{1}, \quad d \eta=-2 \xi_{1} d \xi,
$$

we get

$$
\begin{aligned}
& \left(\int_{|\xi| \leq 1} \frac{\left|\xi_{1}\left(\xi-\xi_{1}\right)\right|^{2 \rho}}{\left(1+\left|\tau_{1}+\xi_{1}^{2}-2 \xi \xi_{1}\right|^{2(1-b)}\right.} d \xi\right)^{1 / 2} \\
\leq & c \frac{\left|\xi_{1}\right|^{2 \rho}}{\left|\xi_{1}\right|^{1 / 2}}\left(\int_{|\eta| \leq\left|\tau_{1}+\xi_{1}^{2}\right|+2\left|\xi_{1}\right|} \frac{d \eta}{(1+|\eta|)^{2(1-b)}}\right)^{1 / 2} \\
\leq & c\left|\xi_{1}\right|^{2 \rho-1 / 2}\left(\left(1+\left|\tau_{1}+\xi_{1}^{2}\right|\right)^{b-1 / 2}+\left|\xi_{1}\right|^{b-1 / 2}\right) .
\end{aligned}
$$

Thus we obtain the following bound for the term in (3.24)

$$
\sup _{\left|\xi_{1}\right| \geq 10} \sup _{\tau_{1}} \frac{\left|\xi_{1}\right|^{2 \rho-1 / 2}}{\left(1+\left|\tau_{1}+\xi_{1}^{2}\right|\right)^{b}}\left(\left(1+\left|\tau_{1}+\xi_{1}^{2}\right|\right)^{b-1 / 2}+\left|\xi_{1}\right|^{b-1 / 2}\right)
$$

which yields the result.

Lemma 3.5. If $\rho=-s \in[0,1 / 4)$, then there exists $b>1 / 2$ such that

$$
\begin{gathered}
\sup _{\left|\xi_{1}\right| \geq 10} \sup _{\tau_{1}} \frac{1}{\left(1+\left|\tau_{1}+\xi_{1}^{2}\right|\right)^{b}} \\
\times\left(\iint_{B_{1}} \frac{\left|\xi_{1}\left(\xi-\xi_{1}\right)\right|^{2 \rho} d \xi d \tau}{(1+|\xi|)^{2 \rho}\left(1+\left|\tau-\tau_{1}-\left(\xi-\xi_{1}\right)^{2}\right|\right)^{2 b}\left(1+\left|\tau-\xi^{2}\right|\right)^{2(1-b)}}\right)^{1 / 2}<\infty
\end{gathered}
$$


where $B_{1}=B_{1}\left(\xi_{1}, \tau_{1}\right)$ is defined as

$$
B_{1}=\left\{\begin{array}{c}
(\xi, \tau) \in \mathbb{R}^{2}:\left|\tau-\tau_{1}-\left(\xi-\xi_{1}\right)^{2}\right| \leq\left|\tau_{1}+\xi_{1}^{2}\right| \\
\left|\tau-\xi^{2}\right| \leq\left|\tau_{1}+\xi_{1}^{2}\right| \\
\left|\xi-\xi_{1}\right| \geq 10 \text { and }|\xi| \geq 1
\end{array}\right\} .
$$

Proof. Arguing as in the previous proof, using that in $B_{1}$

$$
|\eta|=\left|\tau_{1}+\xi_{1}^{2}-2 \xi \xi_{1}\right| \leq 2\left|\tau_{1}+\xi_{1}^{2}\right|
$$

and also using (3.17), we see that the expression in (3.29) can be bounded by

$$
\sup _{\left|\xi_{1}\right| \geq 10} \sup _{\tau}\left(1+\left|\tau_{1}+\xi_{1}^{2}\right|\right)^{2 \rho-1 / 2}
$$

which yields the result.

Lemma 3.6. If $\rho=-s \in[0,1 / 4)$, then there exists $b>1 / 2$ such that

$$
\begin{gathered}
\sup _{\left|\xi_{1}\right| \geq 10} \sup _{\tau_{1}} \frac{1}{\left(1+\left|\tau_{1}-\xi_{1}^{2}\right|\right)^{b}} \\
\times\left(\iint_{C} \frac{\left|\xi_{1}\left(\xi-\xi_{1}\right)\right|^{2 \rho} d \tau d \xi}{(1+|\xi|)^{2 \rho}\left(1+\left|\tau-\tau_{1}+\left(\xi-\xi_{1}\right)^{2}\right|\right)^{2 b}\left(1+\left|\tau-\xi^{2}\right|\right)^{2(1-b)}}\right)^{1 / 2}<\infty
\end{gathered}
$$

where $C=C\left(\xi_{1}, \tau_{1}\right)$ is defined as

$$
C=\left\{\begin{array}{c}
(\xi, \tau) \in \mathbb{R}^{2}:\left|\tau-\tau_{1}+\left(\xi-\xi_{1}\right)^{2}\right| \leq\left|\tau_{1}-\xi_{1}^{2}\right| \\
\left|\tau-\xi^{2}\right| \leq\left|\tau_{1}-\xi_{1}^{2}\right| \\
|\xi| \geq 1 \text { and }\left|\xi-\xi_{1}\right| \geq 10
\end{array}\right\} .
$$

Proof. From (2.3) it follows that

$$
\begin{aligned}
& \int_{\tau \in C} \frac{d \tau}{\left(1+\left|\tau-\tau_{1}+\left(\xi-\xi_{1}\right)^{2}\right|\right)^{2 b}\left(1+\left|\tau-\xi^{2}\right|\right)^{2(1-b)}} \\
\leq & c \frac{\psi\left(\left(\tau_{1}-\xi_{1}^{2}-2 \xi\left(\xi-\xi_{1}\right)\right) / 2\left(\tau_{1}-\xi_{1}^{2}\right)\right)}{\left(1+\left|\tau_{1}-\xi_{1}^{2}-2 \xi\left(\xi-\xi_{1}\right)\right|\right)^{2(1-b)}}
\end{aligned}
$$

since in $C$

$$
\left|\tau_{1}-\xi_{1}^{2}-2 \xi\left(\xi-\xi_{1}\right)\right| \leq\left|\tau-\tau_{1}+\left(\xi-\xi_{1}\right)^{2}\right|+\left|\tau-\xi^{2}\right| \leq 2\left|\tau_{1}-\xi_{1}^{2}\right| .
$$

Also we observe that in $C$ (see (3.17))

$$
\left|\tau_{1}-\xi_{1}^{2}\right| \geq c\left|\xi \xi_{1}\right|
$$

Next we introduce the notation

$$
I(E)=\frac{1}{\left(1+\left|\tau_{1}-\xi_{1}^{2}\right|\right)^{b}}\left(\int_{E} \frac{\left|\xi_{1}\left(\xi-\xi_{1}\right)\right|^{2 \rho}}{(1+|\xi|)^{2 \rho}\left(1+\left|\tau_{1}-\xi_{1}^{2}-2 \xi\left(\xi-\xi_{1}\right)\right|\right)^{2(1-b)}} d \xi\right)^{1 / 2} .
$$


Our aim is to bound $I(D)$ where

$$
D=\left\{\begin{array}{c}
\xi \in C:\left|\tau_{1}-\xi_{1}^{2}-2 \xi\left(\xi-\xi_{1}\right)\right| \leq 2\left|\tau_{1}-\xi_{1}^{2}\right| \\
|\xi| \geq 1 \text { and }\left|\xi-\xi_{1}\right| \geq 10
\end{array}\right\} .
$$

Changing variables

$$
\eta=\tau_{1}-\xi_{1}^{2}-2 \xi\left(\xi-\xi_{1}\right), \quad d \eta=2\left(\xi_{1}-2 \xi\right) d \xi=2 \sqrt{2 \tau_{1}-2 \eta-\xi_{1}^{2}} d \xi
$$

and splitting $D$ into three parts, $D_{1}, D_{2}, D_{3}$ we have, in $D_{1}$, i.e.

$$
D_{1}=\left\{\xi \in D:\left|\xi_{1}\right| \leq 100|\xi|\right\}
$$

from $(3.37),(2.4)$ it follows that

$$
\begin{aligned}
I\left(D_{1}\right) & \leq \frac{1}{\left(1+\left|\tau_{1}-\xi_{1}^{2}\right|\right)^{b-\rho}}\left(\int_{|\eta| \leq 2\left|\tau_{1}-\xi_{1}^{2}\right|} \frac{d \eta}{(1+|\eta|)^{2(1-b)} \sqrt{\left|2 \tau_{1}-2 \eta-\xi_{1}^{2}\right|}}\right)^{1 / 2} \\
& \leq c \frac{\left(1+\left|\tau_{1}-\xi_{1}^{2}\right|\right)^{\rho-1 / 4}}{\left(1+\left|2 \tau_{1}-\xi_{1}^{2}\right|\right)^{1 / 4}}<c^{\prime} .
\end{aligned}
$$

In $D_{2}$, i.e.

$$
D_{2}=\left\{\xi \in D:\left|\xi_{1}\right| \geq 100|\xi| \text { and }\left|\xi_{1}\right| \leq 500\left|\tau_{1}-\xi_{1}^{2}\right|\right\}
$$

we have that the change of variable (3.40) satisfies

$$
\left|\xi_{1}-2 \xi\right| \sim\left|\xi_{1}\right| \text { and } \quad d \eta \simeq \xi_{1} d \xi .
$$

Therefore

$$
\begin{aligned}
I\left(D_{2}\right) & \leq c \frac{\left|\xi_{1}\right|^{2 \rho-1 / 2}}{\left(1+\left|\tau_{1}-\xi_{1}^{2}\right|\right)^{b}}\left(\int_{|\eta| \leq 2\left|\tau_{1}-\xi_{1}^{2}\right|} \frac{d \eta}{(1+|\eta|)^{2(1-b)}}\right)^{1 / 2} \\
& \leq c \frac{\left(1+\left|\tau_{1}-\xi_{1}^{2}\right|\right)^{2 \rho-1 / 2}}{\left(1+\left|\tau_{1}-\xi_{1}^{2}\right|\right)^{b}}\left(1+\left|\tau_{1}-\xi_{1}^{2}\right|\right)^{b-1 / 2} \leq c^{\prime} .
\end{aligned}
$$

Finally, a simple computation shows that

$$
D_{3}=\left\{\xi \in D:\left|\xi_{1}\right| \geq 100|\xi|,\left|\tau_{1}-\xi_{1}^{2}\right| \leq\left|\xi_{1}\right| / 500\right\}
$$

is empty.

Proof of Theorem 1.4(ii). For $N \in \mathbb{Z}^{+}$we define

$$
f_{N}(\xi, \tau)=\psi(\xi-N) \psi\left(\tau-\xi^{2}\right)
$$

and

$$
p_{N}(\xi, \tau)=\psi(\xi+N) \psi\left(\tau+\xi^{2}\right) .
$$

Thus

$$
\left(f_{N} * p_{N}\right)(\xi, \tau) \sim c \chi_{R_{0}}(\xi, \tau)
$$

where $\chi_{A}(\cdot)$ denotes the characteristic function of the set $A$, and $R_{0}$ is the rectangle of dimensions $N \times N^{-1}$ centered at the origin with longest side pointing in the $(1,2 N)$ direction. 
Finally from (3.9) it follows that for $N$ large

$$
N^{2 s} \geq c\left(\int_{|\xi| \leq 1} \int_{|\tau|<1} \chi_{R_{0}}(\xi, \tau) d \xi d \tau\right)^{1 / 2} \geq c N^{-1 / 2} .
$$

Once Theorem 1.2 is available the proof of Theorem 1.6 reduces to that given in section 2 for Theorem 1.5, therefore it will be omitted.

4. Proof of Theorems 1.3, 1.4(iii) AND 1.7

Define

$$
\rho=-s \in[0,3 / 4)
$$

and

$$
f(\xi, \tau)=\left(1+\left|\tau-\xi^{2}\right|\right)^{b}(1+|\xi|)^{-\rho} \hat{F}(\xi, \tau) \in L^{2}\left(\mathbb{R}^{2}\right) .
$$

The argument in section 2 shows that

$$
\|f\|_{L_{\xi}^{2} L_{\tau}^{2}}=\|F\|_{X_{s, b}} .
$$

Thus $B_{3}$ can be expressed in terms of $f$ as

$$
\begin{aligned}
& \left\|B_{3}(F, F)\right\|_{X_{s, b-1}}=\left\|\left(1+\left|\tau-\xi^{2}\right|\right)^{b-1}(1+|\xi|)^{-\rho} \widehat{\bar{F}}\right\|_{L_{\xi}^{2} L_{\tau}^{2}} \\
& =\left\|\left(1+\left|\tau-\xi^{2}\right|\right)^{b-1}(1+|\xi|)^{-\rho}(\widehat{\bar{F}} * \widehat{\bar{F}})\right\|_{L_{\xi}^{2} L_{\tau}^{2}} \\
& =c \| \frac{1}{\left(1+\left|\tau-\xi^{2}\right|\right)^{1-b}(1+|\xi|)^{\rho}} \times \\
& \iint \frac{\bar{f}\left(\xi_{1}-\xi, \tau_{1}-\tau\right)\left(1+\left|\xi-\xi_{1}\right|\right)^{\rho}}{\left(1+\left|\tau-\tau_{1}+\left(\xi-\xi_{1}\right)^{2}\right|\right)^{b}} \frac{\bar{f}\left(-\xi_{1},-\tau_{1}\right)\left(1+\left|\xi_{1}\right|\right)^{\rho}}{\left(1+\left|\tau_{1}+\xi_{1}^{2}\right|\right)^{b}} d \xi_{1} d \tau_{1} \|_{L_{\xi}^{2} L_{\tau}^{2}} .
\end{aligned}
$$

Defining

$$
\begin{gathered}
\mathcal{B}_{3}(f, g, \rho, b)(\xi, \tau)=\frac{1}{\left(1+\left|\tau-\xi^{2}\right|\right)^{1-b}(1+|\xi|)^{\rho}} \times \\
\iint \frac{f\left(\xi_{1}, \tau_{1}\right)\left(1+\left|\xi_{1}\right|\right)^{\rho}}{\left(1+\left|\tau_{1}+\xi_{1}^{2}\right|\right)^{b}} \frac{\left.g\left(\xi-\xi_{1}, \tau-\tau_{1}\right)\left(1+\mid \xi-\xi_{1}\right) \mid\right)^{\rho}}{\left(1+\left|\tau-\tau_{1}+\left(\xi-\xi_{1}\right)^{2}\right|\right)^{b}} d \xi_{1} d \tau_{1}
\end{gathered}
$$

Theorem 1.2 can be rewritten as follows.

Theorem 4.1. Given $\rho=-s \in[0,3 / 4)$ there exists $b \in(1 / 2,1)$ such that

$$
\left\|\mathcal{B}_{3}(f, g, \rho, b)\right\|_{L_{\xi}^{2} L_{\tau}^{2}} \leq c\|f\|_{L_{\xi}^{2} L_{\tau}^{2}}\|g\|_{L_{\xi}^{2} L_{\tau}^{2}} .
$$

The proof of Theorem 4.1 will be a direct consequence of the following lemmas.

Lemma 4.2. If $b \in(1 / 2,1]$, then

$$
\left\|\mathcal{B}_{3}(f, g, 0, b)\right\|_{L_{\xi}^{2} L_{\tau}^{2}} \leq c\|f\|_{L_{\xi}^{2} L_{\tau}^{2}}\|g\|_{L_{\xi}^{2} L_{\tau}^{2}} .
$$


Proof. It suffices to see that

$$
\sup _{\xi, \tau} \frac{1}{\left(1+\left|\tau-\xi^{2}\right|\right)^{1-b}}\left(\iint \frac{d \xi_{1} d \tau_{1}}{\left(1+\left|\tau_{1}+\xi_{1}^{2}\right|\right)^{2 b}\left(1+\left|\tau-\tau_{1}+\left(\xi-\xi_{1}\right)^{2}\right|\right)^{2 b}}\right)^{1 / 2}<\infty .
$$

By changing variables $\left(\xi, \xi_{1}, \tau, \tau_{1}\right) \rightarrow-\left(\xi, \xi_{1}, \tau, \tau_{1}\right)$, the integral in (4.8) is the same as that in (2.12) (Lemma 2.3). Following its proof we obtain the bound

$$
\sup _{\xi, \tau} \frac{1}{\left(1+\left|\tau+\xi^{2}\right|\right)^{1-b}} \frac{1}{\left(1+\left|\tau-\xi^{2} / 2\right|\right)^{1 / 4}},
$$

which yields the result.

In the proof of the following lemmas we use the algebraic relations

$$
\tau-\tau_{1}+\left(\xi-\xi_{1}\right)^{2}+\tau_{1}+\xi_{1}^{2}-\left(\tau-\xi^{2}\right)=\left(\xi-\xi_{1}\right)^{2}+\xi_{1}^{2}+\xi^{2},
$$

and consequently

$$
\max \left\{\left|\tau-\xi^{2}\right| ;\left|\tau_{1}+\xi_{1}^{2}\right| ;\left|\tau-\tau_{1}+\left(\xi-\xi_{1}\right)^{2}\right|\right\} \geq \frac{1}{3}\left(\xi_{1}^{2}+\xi^{2}+\left(\xi-\xi_{1}\right)^{2}\right) .
$$

Lemma 4.3. If $\rho=-s \in(1 / 2,3 / 4)$ and $b \in(1 / 2,5 / 4-s)$, then

$$
\sup _{\xi, \tau} \frac{1}{\left(1+\left|\tau-\xi^{2}\right|\right)^{1-b}} \frac{1}{(1+|\xi|)^{\rho}}
$$

$$
\times\left(\iint_{A_{3}} \frac{\left|\xi_{1}\left(\xi-\xi_{1}\right)\right|^{2 \rho}}{\left(1+\left|\tau_{1}+\xi_{1}^{2}\right|\right)^{2 b}\left(1+\left|\tau-\tau_{1}+\left(\xi-\xi_{1}\right)^{2}\right|\right)^{2 b}} d \tau_{1} d \xi_{1}\right)^{1 / 2}<\infty
$$

where $A_{3}=A_{3}(\xi, \tau)$ is defined as

$$
A_{3}=\left\{\left(\xi_{1}, \tau_{1}\right):\left|\tau-\tau_{1}+\left(\xi-\xi_{1}\right)^{2}\right| \leq\left|\tau_{1}+\xi_{1}^{2}\right| \leq\left|\tau-\xi^{2}\right|\right\} .
$$

Proof. Changing $\left(\tau, \tau_{1}\right)$ by $-\left(\tau, \tau_{1}\right)$ and following the argument in the proof of Lemma 2.4 (2.22) one obtains the following bound for (4.12)

$$
\sup _{\xi, \tau} \frac{1}{(1+|\xi|)^{\rho}} \frac{\left(1+\left|\tau+\xi^{2}\right|\right)^{\rho+b-1}}{\left(1+\left|\tau-\xi^{2} / 2\right|\right)^{1 / 4}}
$$

which yields the result.

Lemma 4.4. If $\rho=-s \in(1 / 2,3 / 4]$, then there exists $b>1 / 2$ such that

$$
\begin{gathered}
\sup _{\left|\xi_{1}\right| \geq 1} \sup _{\tau_{1}} \frac{1}{\left(1+\left|\tau_{1}+\xi_{1}^{2}\right|\right)^{b}} \\
\times\left(\iint_{B_{3}} \frac{\left|\xi_{1}\left(\xi-\xi_{1}\right)\right|^{2 \rho}}{(1+|\xi|)^{\rho}\left(1+\left|\tau-\xi^{2}\right|\right)^{2(1-b)}\left(1+\left|\tau-\tau_{1}+\left(\xi-\xi_{1}\right)^{2}\right|\right)^{2 b}} d \tau d \xi\right)^{1 / 2}<\infty
\end{gathered}
$$

where $B_{3}=B_{3}\left(\xi_{1}, \tau_{1}\right)$ is defined as

$$
B_{3}=\left\{\begin{array}{c}
(\xi, \tau) \in \mathbb{R}^{2}:\left|\tau-\tau_{1}+\left(\xi-\xi_{1}\right)^{2}\right| \leq\left|\tau_{1}+\xi_{1}^{2}\right| \\
\text { and }\left|\tau-\xi^{2}\right| \leq\left|\tau_{1}+\xi_{1}^{2}\right|
\end{array}\right\} .
$$


Proof. Following an argument similar to that used in the proof of Lemma 2.5, we bound (4.15) by

$$
\sup _{\left|\xi_{1}\right| \geq 1, \tau_{1}} \frac{1}{\left(1+\left|\tau_{1}+\xi_{1}^{2}\right|\right)^{b}}\left(\int_{D^{\prime}} \frac{\left|\xi_{1}\left(\xi-\xi_{1}\right)\right|^{2 \rho} d \xi}{(1+|\xi|)^{2 \rho}\left(1+\left|\tau_{1}-\xi^{2}-\left(\xi-\xi_{1}\right)^{2}\right|\right)^{2(1-b)}}\right)^{1 / 2}
$$

with $D^{\prime}=D^{\prime}(\xi)$,

$$
D^{\prime}=\left\{\xi \in \mathbb{R}:\left|\tau_{1}-\xi^{2}-\left(\xi-\xi_{1}\right)^{2}\right| \leq 2\left|\tau_{1}+\xi_{1}^{2}\right|\right\} .
$$

First we consider the subset of $D^{\prime}$

$$
D_{1}^{\prime}=\left\{\xi \in D^{\prime}: \xi^{2}+\left(\xi-\xi_{1}\right)^{2}+\xi_{1}^{2} \leq\left|\tau_{1}+\xi_{1}^{2}\right| / 2\right\} .
$$

In this domain it follows that

$$
\begin{aligned}
& \frac{1}{\left(1+\left|\tau_{1}+\xi_{1}^{2}\right|\right)^{b}}\left(\int_{D_{1}^{\prime}} \frac{\left|\xi_{1}\left(\xi-\xi_{1}\right)\right|^{2 \rho}}{(1+|\xi|)^{2 \rho}\left(1+\left|\tau_{1}-\xi^{2}-\left(\xi-\xi_{1}\right)^{2}\right|\right)^{2(1-b)}} d \xi\right)^{1 / 2} \\
& \leq \frac{\left(1+\left|\tau_{1}+\xi_{1}^{2}\right|\right)^{\rho}}{\left(1+\left|\tau_{1}+\xi_{1}^{2}\right|\right)}\left(\int \frac{d \xi}{(1+|\xi|)^{2 \rho}}\right)^{1 / 2}<\infty .
\end{aligned}
$$

Next we consider the remaining part of $D_{1}$, i.e.

$$
D_{2}^{\prime}=\left\{\xi \in D^{\prime}:\left|\tau_{1}+\xi_{1}^{2}\right| / 2 \leq \xi^{2}+\left(\xi-\xi_{1}\right)^{2}+\xi_{1}^{2} \leq 3\left|\tau_{1}+\xi_{1}^{2}\right|\right\} .
$$

We split $D_{2}^{\prime}$ into three pieces $D_{2,1}^{\prime}, D_{2,2}^{\prime}$ and $D_{2,3}^{\prime}$. In $D_{2,1}^{\prime}$, i.e.

$$
D_{2,1}^{\prime}=\left\{\xi \in D_{2}^{\prime}:|\xi| / 4 \leq\left|\xi_{1}\right| \leq 100|\xi|\right\}
$$

combining the change of variables

$$
\eta=\tau_{1}-\xi^{2}-\left(\xi-\xi_{1}\right)^{2}, \quad d \eta=-2\left(\xi_{1}-2 \xi\right) d \xi, \quad\left|\xi_{1}-2 \xi\right| \simeq \sqrt{\left.\left(2 \tau_{1}-\xi^{2}\right) / 2-\eta\right),}
$$

so that since $|\xi| \simeq\left|\xi_{1}\right|$ we write

$$
\begin{aligned}
& \frac{1}{\left(1+\left|\tau_{1}+\xi_{1}^{2}\right|\right)^{b}}\left(\int_{D_{2,1}^{\prime}} \frac{\left|\xi_{1}\left(\xi-\xi_{1}\right)\right|^{2 \rho}}{(1+|\xi|)^{2 \rho}\left(1+\left|\tau_{1}-\xi^{2}-\left(\xi-\xi_{1}\right)^{2}\right|\right)^{2(1-b)}} d \xi\right)^{1 / 2} \\
\leq & \frac{c\left(1+\left|\tau_{1}+\xi_{1}^{2}\right|\right)^{\rho / 2}}{\left(1+\left|\tau_{1}+\xi_{1}^{2}\right|\right)^{b}}\left(\frac{\int_{|\eta| \leq 3\left|\tau_{1}+\xi_{1}^{2}\right|} d \eta}{\sqrt{\left.\left(2 \tau_{1}-\xi^{2}\right) / 2-\eta\right)(1+|\eta|)^{2(1-b)}}}\right)^{1 / 2} \\
\leq & c \frac{\left(1+\left|\tau_{1}+\xi_{1}^{2}\right|\right)^{\rho / 2-1 / 4}}{\left(1+\left|2 \tau_{1}-\xi_{1}^{2}\right|\right)^{1 / 4}} \leq c .
\end{aligned}
$$

In $D_{2,2}^{\prime}$, i.e.

$$
D_{2,2}^{\prime}=\left\{\xi \in D_{2}^{\prime}: 1 \leq\left|\xi_{1}\right| \leq|\xi| / 4\right\},
$$

one has in (4.23) that $\left|\xi_{1}\right| /|\xi|<1$, hence, the argument in (4.24) extends to this case.

Finally we consider

$$
D_{2,3}^{\prime}=\left\{\xi \in D_{1}^{\prime}: 100|\xi| \leq\left|\xi_{1}\right|\right\} .
$$


In this region, the change of variable (4.23) satisfies

$$
d \eta=-2\left(2 \xi-\xi_{1}\right) d \xi \simeq\left|\xi_{1}\right| d \xi .
$$

An argument similar to that in (4.24) gives the bound

$$
\frac{\left|\xi_{1}\right|^{2 \rho-1 / 2}}{\left(1+\left|\tau_{1}+\xi_{1}^{2}\right|\right)^{1 / 2}} \leq c\left(1+\left|\tau_{1}+\xi_{1}^{2}\right|\right)^{\rho-3 / 4}
$$

Proof of Theorem 1.4(iii). For $N \in \mathbb{Z}^{+}$define

$$
f_{N}(\xi, \tau)=\psi(\xi-N) \psi\left(\tau+\xi^{2}\right)
$$

and

$$
g_{N}(\xi, \tau)=\psi(\xi+N) \psi\left(\tau+\xi^{2}\right)
$$

Since for large $N$

$$
\left(f_{N} * g_{N}\right)(\xi, \tau) \geq \frac{c}{N} \psi\left(-2 N^{2}-\tau / N\right) \psi(\xi)
$$

(4.6) implies that

$$
\frac{1}{N^{2(1-b)}} \frac{N^{2 \rho}}{N^{1 / 2}} \leq c
$$

On the other hand, if

$$
h_{N}(\xi, \tau)=\psi(\xi+N) \psi\left(\tau-\xi^{2}\right)
$$

it is easy to see that

$$
\left(f_{N} * h_{N}\right)(\xi, \tau) \cong c \chi_{R}(\xi, \tau)
$$

where $R$ is the rectangle of dimensions $c N \times N^{-1}$ centered at the origin with its longest side pointing in the $(1,2 N)$ direction.

Thus (4.6) shows that

$$
\frac{1}{N^{1-b}} \frac{N^{2 \rho}}{N^{2 b}} \leq c .
$$

Collecting (4.32), (4.35) and letting $N$ tend to infinity we obtain that

$$
\rho \leq \min \left\{\frac{5}{4}-b ; \frac{b}{2}+\frac{1}{2}\right\},
$$

which yields the result.

Finally, we remark that once Theorem 1.3 has been established, the proof of Theorem 1.7 follows the argument used in Section 2 for proving Theorem 1.5, therefore it will be omitted.

\section{Proof of Theorems $1.8-1.10$}

For $\rho=-s \in[0,1 / 2]$ and $F \in Y_{s, b}$ define

$$
f(n, \tau)=\left(1+\left|\tau-n^{2}\right|\right)^{b}(1+|n|)^{s} \hat{F}(n, \tau) \in \ell_{n}^{2} L_{\tau}^{2} .
$$

Thus

$$
\|f\|_{\ell_{n}^{2} L_{\tau}^{2}}=\|F\|_{Y_{s, b}}
$$


and (1.27) can be rewritten in terms of $f$

$$
\begin{aligned}
& \left\|B_{1}(F, F)\right\|_{Y_{s, b-1}}=\left\|\left(1+\left|\tau-n^{2}\right|\right)^{b-1}(1+|n|)^{s} \widehat{F^{2}}\right\|_{\ell_{n}^{2} L_{\tau}^{2}} \\
= & \left\|\left(1+\left|\tau-n^{2}\right|\right)^{b-1}(1+|n|)^{s}(\hat{F} * \hat{F})\right\|_{\ell_{n}^{2} L_{\tau}^{2}} \\
= & \| \frac{1}{\left(1+\left|\tau-n^{2}\right|\right)^{1-b}(1+|n|)^{\rho}} \\
\times & \sum_{n_{1}} \int \frac{f\left(n_{1}, \tau_{1}\right)\left(1+\left|n_{1}\right|\right)^{\rho}}{\left(1+\left|\tau_{1}-n_{1}^{2}\right|\right)^{b}} \frac{f\left(n-n_{1}, \tau-\tau_{1}\right)\left(1+\left|n-n_{1}\right|\right)^{\rho}}{\left(1+\left|\tau-\tau_{1}-\left(n-n_{1}\right)^{2}\right|\right)^{b}} d \tau_{1} \|_{\ell_{n}^{2} L_{\tau}^{2}} .
\end{aligned}
$$

Defining

$$
\begin{aligned}
& \mathcal{B}_{1}\left(f, g, \rho, b, b^{\prime}\right)(n, \tau)=\frac{1}{\left(1+\left|\tau-n^{2}\right|\right)^{1-b}(1+|n|)^{\rho}} \\
\times & \sum_{n_{1}} \int \frac{f\left(n_{1}, \tau_{1}\right)\left(1+\left|n_{1}\right|\right)^{\rho}}{\left(1+\left|\tau_{1}-n_{1}^{2}\right|\right)^{b^{\prime}}} \frac{g\left(n-n_{1}, \tau-\tau_{1}\right)\left(1+\left|n-n_{1}\right|\right)^{\rho}}{\left(1+\left|\tau-\tau_{1}-\left(n-n_{1}\right)^{2}\right|\right)^{b^{\prime}}} d \tau_{1}
\end{aligned}
$$

we restate Theorem 1.8.

Theorem 5.1. If $\rho=-s \in[0,1 / 2]$, then

$$
\left\|\mathcal{B}_{1}(f, g, \rho, 1 / 2,1 / 2)\right\|_{\ell_{n}^{2} L_{\tau}^{2}} \leq c\|f\|_{\ell_{n}^{2} L_{\tau}^{2}}\|g\|_{\ell_{n}^{2} L_{\tau}^{2}} .
$$

As a consequence of the proof of Theorem 5.1 we have

Corollary 5.2. If $\rho=-s \in[0,1 / 2)$ and $1-b, b^{\prime} \geq \rho$ with $1-b, b^{\prime}>3 / 8$, then

$$
\left\|\mathcal{B}_{1}\left(f, g, \rho, b, b^{\prime}\right)\right\|_{\ell_{n}^{2} L_{\tau}^{2}} \leq c\|f\|_{\ell_{n}^{2} L_{\tau}^{2}}\|g\|_{\ell_{n}^{2} L_{\tau}^{2}} .
$$

The following lemmas will be needed in the proof of Theorems 1.8-1.9.

Lemma 5.3. If $\gamma>1 / 2$, then

$$
\sup _{(n, \tau) \in \mathbb{Z} \times \mathbb{R}} \sum_{n_{1}=-\infty}^{\infty} \frac{1}{\left(1+\left|\tau \pm n_{1}\left(n-n_{1}\right)\right|\right)^{\gamma}}<\infty .
$$

Proof. We rewrite (5.7)

$$
\sum_{n_{1}=-\infty}^{\infty} \frac{1}{\left(1+\left|\tau \pm n_{1}\left(n-n_{1}\right)\right|\right)^{\gamma}}=\sum_{n_{1}} \frac{1}{\left(1+\left|\left(n_{1}-\alpha^{ \pm}\right)\left(n_{1}-\beta^{ \pm}\right)\right|\right)^{\gamma}}
$$

where $\alpha=\alpha^{ \pm}(n, \tau), \beta=\beta^{ \pm}(n, \tau)$ are the roots of the polynomial

$$
\tau \pm\left(n_{1}\left(n-n_{1}\right)=0, \quad \text { i.e. } \quad \tau \pm n_{1}\left(n-n_{1}\right)=\left(n_{1}-\alpha^{ \pm}\right)\left(n_{1}-\beta^{ \pm}\right) .\right.
$$

There are at most $10 n_{1}^{\prime}$ 's such that $\left|n_{1}-\alpha\right| \leq 2$ or $\left|n_{1}-\beta\right| \leq 2$. The remaining $n_{1}$ 's satisfy

$$
\left(1+\left|\left(n_{1}-\alpha\right)\left(n_{1}-\beta\right)\right|\right) \geq \frac{1}{2}\left(1+\left|n_{1}-\alpha\right|\right)\left(1+\left|n_{1}-\beta\right|\right) .
$$

Hence, applying the Cauchy-Schwarz inequality in (5.7) we obtain the desired result. 
In the proof of the following lemmas the following algebraic relation will be used

$$
\tau-n^{2}-\left(\tau_{1}-n_{1}^{2}\right)-\left(\tau-\tau_{1}-\left(n-n_{1}\right)^{2}\right)=-2 n_{1}\left(n-n_{1}\right) .
$$

In particular, this guarantees that

$$
\max \left\{\left|\tau-n^{2}\right| ;\left|\tau_{1}-n_{1}^{2}\right| ;\left|\tau-\tau_{1}-\left(n-n_{1}\right)^{2}\right|\right\} \geq \frac{2}{3}\left|n_{1}\left(n-n_{1}\right)\right| .
$$

Lemma 5.4. If $\rho=-s \in[0,1 / 2]$, then

$$
\sup _{(n, \tau) \in \mathbb{Z} \times \mathbb{R}} \frac{1}{\left(1+\left|\tau-n^{2}\right|\right)^{1 / 2}} \frac{1}{(1+|n|)^{\rho}}
$$

$$
\times\left(\sum_{n_{1} \in A} \int_{\tau_{1} \in A} \frac{\left(1+\left|n_{1}\right|\right)^{2 \rho}\left(1+\left|n-n_{1}\right|\right)^{2 \rho}}{\left(1+\left|\tau_{1}-n_{1}^{2}\right|\right)\left(1+\left|\tau-\tau_{1}-\left(n-n_{1}\right)^{2}\right|\right)}\right)^{1 / 2}<\infty
$$

where $A=A(n, \tau)$ is defined as

$$
A=\left\{\left(n_{1}, \tau_{1}\right) \in \mathbb{Z} \times \mathbb{R}:\left|\tau-\tau_{1}-\left(n-n_{1}\right)^{2}\right| \leq\left|\tau_{1}-n_{1}\right| \leq\left|\tau-n^{2}\right|\right\} .
$$

Proof. It suffices to consider the extremal cases, $\rho=0$ and $\rho=1 / 2$. If $\rho=0$, changing variables

$$
\theta_{1}=\tau_{1}-n_{1}^{2}
$$

it follows that

$$
\begin{aligned}
& \int_{\tau_{1} \in A} \frac{d \tau_{1}}{\left(1+\left|\tau_{1}-n_{1}^{2}\right|\right)\left(1+\left|\tau-\tau_{1}-\left(n-n_{1}\right)^{2}\right|\right)} \\
= & \int \frac{d \theta_{1}}{\left(1+\left|\theta_{1}\right|\right)\left(1+\left|\theta_{1}-\left(\tau-n^{2}+2 n_{1}\left(n-n_{1}\right)\right)\right|\right)} \\
\leq & \frac{\ln \left(2+\left|\tau-n^{2}+2 n_{1}\left(n-n_{1}\right)\right|\right)}{\left(1+\left|\tau-n^{2}+2 n_{1}\left(n-n_{1}\right)\right|\right)} .
\end{aligned}
$$

Thus we have bound the term in (5.13) by

$$
\sup _{(n, \tau) \in \mathbb{Z} \times \mathbb{R}}\left(\sum_{n_{1}} \frac{\ln \left(2+\left|\tau-n^{2}+2 n_{1}\left(n-n_{1}\right)\right|\right)}{1+\left|\tau-n^{2}+2 n_{1}\left(n-n_{1}\right)\right|}\right)^{1 / 2} .
$$

Hence (5.7) completes the proof.

We observe that the factor $\left(1+\left|\tau-n^{2}\right|\right)^{-1 / 2}$ in (5.13) has not been used in the proof.

If $\rho=1 / 2$, the bound for the values $n_{1}=0$ and $n_{1}=n$ follows from the previous case. Now restricting the sum in (5.13) to the $n_{1}$ 's such that $n_{1} \neq 0$ and $n_{1} \neq n$ from (5.12) it follows that

$$
\left(1+\left|\tau-n^{2}\right|\right) \geq \frac{1}{3}\left(1+\left|n_{1}\right|\right)\left(1+\left|n-n_{1}\right|\right),
$$

which reduces the proof to the previous case $\rho=0$. 
Lemma 5.5. If $\rho=-s \in[0,-1 / 2]$, then

$$
\begin{gathered}
\sup _{\left(n_{1}, \tau_{1}\right) \in \mathbb{Z} \times \mathbb{R}} \frac{1}{\left(1+\left|\tau_{1}-n_{1}^{2}\right|\right)^{1 / 2}} \\
\times\left(\sum_{n \in D} \int_{\tau \in D} \frac{\left(1+\left|n_{1}\right|\right)^{2 \rho}\left(1+\left|n-n_{1}\right|\right)^{2 \rho}}{(1+|n|)^{2 \rho}\left(1+\left|\tau-n^{2}\right|\right)\left(1+\left|\tau-\tau_{1}-\left(n-n_{1}\right)^{2}\right|\right)} d \tau\right)^{1 / 2}<\infty,
\end{gathered}
$$

where $D=D\left(n_{1}, \tau_{1}\right)$ is defined as

$D=\left\{(n, \tau) \in \mathbb{Z} \times \mathbb{R}:\left|\tau-\tau_{1}-\left(n-n_{1}\right)^{2}\right| \leq\left|\tau_{1}-n_{1}^{2}\right|\right.$ and $\left.\left|\tau-n^{2}\right| \leq\left|\tau_{1}-n_{1}^{2}\right|\right\}$.

Proof. It is similar to the proof of the previous lemma, hence it will be omitted.

Proof of Theorem 5.1. From (5.13)-(5.14) and symmetry it follows that

$$
\begin{gathered}
\left\|\mathcal{B}_{1}(f, g, \rho, 1 / 2,1 / 2)\right\|_{\ell_{n}^{2} L_{\tau}^{2}(A)} \\
\leq c \sup _{n, \tau} \frac{1}{\left(1+\left|\tau-n^{2}\right|\right)^{1 / 2}} \frac{1}{(1+|n|)^{\rho}} \\
\times\left(\sum_{n_{1} \in A} \int_{\tau_{1} \in A} \frac{\left(1+\left|n_{1}\right|\right)^{2 \rho}\left(1+\left|n-n_{1}\right|\right)^{2 \rho}}{\left(1+\left|\tau_{1}-n_{1}^{2}\right|\right)\left(1+\left|\tau-\tau_{1}-\left(n-n_{1}\right)^{2}\right|\right)} d \tau_{1}\right)^{1 / 2} \\
\times\|f\|_{\ell_{n}^{2} L_{\tau}^{2}}\|g\|_{\ell_{n}^{2} L_{\tau}^{2}} \leq c\|f\|_{\ell_{n}^{2} L_{\tau}^{2}}\|g\|_{\ell_{n}^{2} L_{\tau}^{2} .}
\end{gathered}
$$

Also by duality and (5.20)

$$
\begin{aligned}
& \left\|\mathcal{B}_{1}(f, g, \rho, 1 / 2,1 / 2)\right\|_{\ell_{n}^{2} L_{\tau}^{2}(D)} \\
= & \sup _{\|h\|_{\ell_{n}^{2} L_{\tau}^{2} \leq 1}} \sum_{n} \int\left|\mathcal{B}_{1}(f, g, \rho, 1 / 2,1 / 2)(n, \tau) \chi_{D} h(n, \tau)\right| d \tau \\
= & \sup _{\|h\|_{\ell_{n}^{2} L_{\tau}^{2} \leq 1}} \sum_{n_{1}} \int\left|\mathcal{B}_{1}^{*}\left(h \chi_{D}, g, \rho, 1 / 2\right)\left(n_{1}, \tau_{1}\right) f\left(n_{1}, \tau_{1}\right)\right| d \tau_{1}
\end{aligned}
$$

where

$$
\begin{gathered}
\mathcal{B}_{1}^{*}\left(p, q, \rho, b, b^{\prime}\right)\left(n_{1}, \tau_{1}\right)=\frac{\left(1+\left|n_{1}\right|\right)^{\rho}}{\left(1+\left|\tau_{1}-n_{1}^{2}\right|\right)^{b^{\prime}}} \\
\times \sum_{n \in D} \int_{\tau \in D} \frac{p(n, \tau)}{(1+|n|)^{\rho}\left(1+\left|\tau-n^{2}\right|\right)^{1-b}} \frac{q\left(n-n_{1}, \tau-\tau_{1}\right)\left(1+\left|n-n_{1}\right|\right)^{\rho}}{\left(1+\left|\tau-\tau_{1}-\left(n-n_{1}\right)^{2}\right|\right)^{b^{\prime}}} d \tau .
\end{gathered}
$$

The argument in (5.21) combined with (5.13) shows that

$$
\left\|\mathcal{B}_{1}^{*}(p, q, \rho, 1 / 2,1 / 2)\right\|_{\ell_{n}^{2} L_{\tau}^{2}} \leq c\|p\|_{\ell_{n}^{2} L_{\tau}^{2}}\|q\|_{\ell_{n}^{2} L_{\tau}^{2}} .
$$

Collecting (5.21)-(5.24) we obtain the desired result. 
Proof of Theorem 1.10(i). For $N \in \mathbb{Z}$ define

$$
h_{N}(n, \tau)=a_{n} \chi\left(\left(\tau-n^{2}\right) / 2\right), \quad \text { with } \quad a_{n}= \begin{cases}1, & n=N, \\ 0, & \text { elsewhere }\end{cases}
$$

and

$$
g_{N}(n, \tau)=b_{n} \chi\left(\left(\tau-n^{2}\right) / 2\right), \quad \text { with } \quad b_{n}= \begin{cases}1, & n=1-N, \\ 0, & \text { elsewhere },\end{cases}
$$

where $\chi(\cdot)$ denotes the characteristic function of the interval $[-1,1]$. Thus

$$
a_{n_{1}} b_{n-n_{1}} \neq 0 \text { if and only if } n_{1}=N \text { and } n=1
$$

and consequently for $N$ large

$$
\begin{aligned}
\int \chi\left(\left(\tau_{1}-n_{1}^{2}\right) / 2\right) \chi\left(\left(\tau-\tau_{1}-\left(n-n_{1}\right)^{2}\right) / 2\right) d \tau & \cong \chi\left(\tau-\left(n-n_{1}\right)^{2}-n_{1}^{2}\right) \\
& \cong \chi(\tau-1+2 N(N-1)) .
\end{aligned}
$$

Therefore from the definition in (5.4)

$$
\mathcal{B}_{1}\left(h_{N}, g_{N}, \rho, b, b^{\prime}\right)(1, \tau) \geq c \frac{N^{2 \rho}}{(1+|\tau-1|)^{1-b}} \chi(\tau-1+2 N(N-1)) .
$$

Hence, (5.6) implies that

$$
\frac{N^{2 \rho}}{N^{2(1-b)}} \leq c
$$

Now we define

$$
p_{N}(n, \tau)=a_{n} \chi\left(\left(\tau-n^{2}\right) / 2\right), \quad \text { with } \quad a_{n}= \begin{cases}1, & n=1 \\ 0, & \text { elsewhere }\end{cases}
$$

and

$$
g_{N}(n, \tau)=b_{n} \chi\left(\left(\tau-n^{2}\right) / 2\right), \quad \text { with } \quad b_{n}= \begin{cases}1, & n=N-1 \\ 0, & \text { elsewhere }\end{cases}
$$

Thus

$$
a_{n_{1}} b_{n_{1}-n} \neq 0 \text { if and only if } n_{1}=N \text { and } n=1 \text {, }
$$

and

$$
\begin{aligned}
\int \chi\left(\left(\tau-n^{2}\right) / 2\right) \chi\left(\left(\tau-\tau_{1}-\left(n-n_{1}\right)^{2}\right) / 2\right) d \tau & \sim \chi\left(\tau_{1}+\left(n-n_{1}\right)^{2}+n^{2}\right) \\
& \sim \chi\left(\tau_{1}+N^{2}-2 N\right) .
\end{aligned}
$$

Hence, using the definition in (5.23)

$$
\mathcal{B}_{1}^{*}\left(p_{N}, g_{N}, \rho, b, b^{\prime}\right)\left(N, \tau_{1}\right) \geq c \frac{N^{2 \rho}}{\left(1+\left|\tau_{1}-n_{1}^{2}\right|\right)^{b^{\prime}}} \chi\left(\tau_{1}+N^{2}-2 N\right) .
$$

Then (5.6) affirms that

$$
\frac{N^{2 \rho}}{N^{2 b^{\prime}}} \leq c .
$$

Combining (5.30), (5.36) the results in Theorem 1.10(i) follow. 
Now we turn our attention to Theorem 1.9. As in the proof of Theorem 1.8 defining

$$
\begin{gathered}
\mathcal{B}_{3}\left(f, g, \rho, b, b^{\prime}\right)(n, \tau)=\frac{1}{\left(1+\left|\tau-n^{2}\right|\right)^{1-b}(1+|n|)^{\rho}} \\
\times \sum_{n_{1}} \int \frac{f\left(n_{1}, \tau_{1}\right)\left(1+\left|n_{1}\right|\right)^{\rho}}{\left(1+\left|\tau_{1}+n_{1}^{2}\right|\right)^{b^{\prime}}} \frac{g\left(n-n_{1}, \tau-\tau_{1}\right)\left(1+\left|n-n_{1}\right|\right)^{\rho}}{\left(1+\left|\tau-\tau_{1}+\left(n-n_{1}\right)^{2}\right|\right)^{b^{\prime}}} d \tau
\end{gathered}
$$

we restate Theorem 1.9.

Theorem 5.6. If $\rho=-s \in[0,1 / 2]$, then

$$
\left\|\mathcal{B}_{3}(f, g, \rho, 1 / 2,1 / 2)\right\|_{\ell_{n}^{2} L_{\tau}^{2}} \leq c\|f\|_{\ell_{n}^{2} L_{\tau}^{2}}\|g\|_{\ell_{n}^{2} L_{\tau}^{2}} .
$$

From the proof of Theorem 5.6 one can obtain the following result.

Corollary 5.7. If $\rho=-s \in[0,1 / 2)$ and $1-b, b^{\prime} \geq \rho$ with $1-b, b^{\prime}>3 / 8$, then

$$
\left\|\mathcal{B}_{3}\left(f, g, \rho, b, b^{\prime}\right)\right\|_{\ell_{n}^{2} L_{\tau}^{2}} \leq c\|f\|_{\ell_{n}^{2} L_{\tau}^{2}}\|g\|_{\ell_{n}^{2} L_{\tau}^{2}} .
$$

We shall use the algebraic relation

$$
\tau-n^{2}-\left(\tau_{1}+n_{1}^{2}\right)-\left(\tau-\tau_{1}+\left(n-n_{1}\right)^{2}\right)=-n^{2}-n_{1}^{2}-\left(n-n_{1}\right)^{2} .
$$

In particular, this implies that

$\max \left\{\left|\tau-n^{2}\right| ;\left|\tau_{1}+n_{1}^{2}\right| ;\left|\tau-\tau_{1}+\left(n-n_{1}\right)^{2}\right|\right\} \geq n^{2}+n_{1}^{2}+\left(n-n_{1}\right)^{2} \geq \frac{1}{2} n\left(n-n_{1}\right)$.

The proof of Theorem 5.1 is a direct consequence of the following two lemmas, whose proofs we omit since they are similar to those given for Lemmas 5.4 and 5.5.

Lemma 5.8. If $\rho=-s \in[0,1 / 2]$, then

$$
\sup _{(n, \tau) \in \mathbb{Z} \times \mathbb{R}} \frac{1}{\left(1+\left|\tau-n^{2}\right|\right)^{1 / 2}} \frac{1}{(1+|n|)^{\rho}}
$$

$$
\times\left(\sum_{\left(n_{1}, \tau_{1}\right) \in A_{1}} \int \frac{\left(1+\left|n_{1}\right|\right)^{2 \rho}\left(1+\left|n-n_{1}\right|\right)^{2 \rho}}{\left(1+\left|\tau_{1}+n_{1}^{2}\right|\right)\left(1+\left|\tau-\tau_{1}+\left(n-n_{1}\right)^{2}\right|\right)} d \tau_{1}\right)^{1 / 2}<\infty
$$

where

$$
A_{1}=A_{1}(n, \tau)=\left\{\left(n_{1}, \tau_{1}\right) \in \mathbb{Z} \times \mathbb{R}:\left|\tau-\tau_{1}+\left(n-n_{1}\right)^{2}\right| \leq\left|\tau_{1}-n_{1}^{2}\right| \leq\left|\tau-n^{2}\right|\right\} .
$$

Lemma 5.9. If $\rho=-s \in[0,1 / 2]$, then

$$
\begin{gathered}
\sup _{\left(n_{1}, \tau_{1}\right) \in \mathbb{Z} \times \mathbb{R}} \frac{1}{\left(1+\left|\tau_{1}+n_{1}^{2}\right|\right)^{1 / 2}} \\
\times\left(\sum_{(n, \tau) \in D_{1}} \int \frac{\left(1+\left|n_{1}\right|\right)^{2 \rho}\left(1+\left|n-n_{1}\right|\right)^{2 \rho}}{(1+|n|)^{2 \rho}\left(1+\left|\tau-n^{2}\right|\right)\left(1+\left|\tau-\tau_{1}-\left(n-n_{1}\right)^{2}\right|\right)} d \tau\right)^{1 / 2}<\infty
\end{gathered}
$$


QUADRATIC FORMS FOR THE 1-D SEMILINEAR SCHRÖDINGER EQUATION 3351 where

$$
D_{1}=D_{1}\left(n_{1}, \tau_{1}\right)=\left\{\begin{array}{c}
(n, \tau) \in \mathbb{Z} \times \mathbb{R}:\left|\tau-\tau_{1}+\left(n-n_{1}\right)^{2}\right| \leq\left|\tau_{1}+n_{1}^{2}\right| \\
\text { and }\left|\tau-n^{2}\right| \leq\left|\tau_{1}+n_{1}^{2}\right|
\end{array}\right\} .
$$

Proof of Theorem 1.10(ii). For $N \in \mathbb{Z}$ define

$$
f_{N}(n, \tau)=a_{n} \chi\left(\left(\tau+n^{2}\right) / 2\right), \quad \text { with } \quad a_{n}= \begin{cases}1, & n=N \\ 0, & \text { elsewhere }\end{cases}
$$

and

$$
g_{N}(n, \tau)=b_{n} \chi\left(\left(\tau+n^{2}\right) / 2\right), \quad \text { with } \quad b_{n}= \begin{cases}1, & n=1-N, \\ 0, & \text { elsewhere }\end{cases}
$$

with $\chi(\cdot)$ denoting the characteristic function of the interval $[-1,1]$. Thus

$$
a_{n_{1}} b_{n-n_{1}} \neq 0 \text { if and only if } n_{1}=N \text { and } n=1,
$$

and for $N$ large

$$
\begin{aligned}
\int \chi\left(\left(\tau_{1}+n_{1}^{2}\right) / 2\right) \chi\left(\left(\tau-\tau_{1}+\left(n-n_{1}\right)^{2}\right) / 2\right) d \tau_{1} & \cong \chi\left(\tau+\left(n-n_{1}\right)^{2}+n_{1}^{2}\right) \\
& \cong \chi(\tau+1+2 N(N-1)) .
\end{aligned}
$$

From the definition (5.37) it follows that

$$
\mathcal{B}_{3}\left(f_{n}, g_{N}, \rho, b, b^{\prime}\right)(1, \tau) \geq c \frac{N^{2 \rho}}{(1+|\tau+1|)^{1-b}} \chi(\tau+1+2 N(N-1)),
$$

and from (5.38)

$$
\frac{N^{2 \rho}}{N^{2(1-b)}} \leq c
$$

A similar argument with

$$
p_{N}(n, \tau)=a_{n} \chi\left(\left(\tau+n^{2}\right) / 2\right), \quad \text { with } \quad a_{n}= \begin{cases}1, & n=1, \\ 0, & \text { elsewhere }\end{cases}
$$

and

$$
q_{N}(n, \tau)=b_{n} \chi\left(\left(\tau+n^{2}\right) / 2\right), \quad \text { with } \quad b_{n}= \begin{cases}1, & n=N-1 \\ 0, & \text { elsewhere }\end{cases}
$$

shows that

$$
\mathcal{B}_{3}^{*}\left(p_{N}, q_{N}, \rho, b, b^{\prime}\right)\left(N, \tau_{1}\right) \geq c \frac{N^{2 \rho}}{\left(1+\left|\tau_{1}+n_{1}\right|\right)^{b^{\prime}}} \chi\left(\tau_{1}-\left(n-n_{1}\right)^{2}+n^{2}\right)
$$

where $\mathcal{B}_{3}^{*}$ is defined similarly as $\mathcal{B}_{1}^{*}$ in $(5.23)$, and by (5.38) that

$$
\frac{N^{2 \rho}}{N^{2 b^{\prime}}} \leq c \text {. }
$$

Combining (5.51)-(5.55) we obtain the desired result. 
Proof of Theorem 1.10(iii). We shall prove that for any $\rho=-s>0$ and $b \in \mathbb{R}$

$$
\begin{gathered}
\mathcal{B}_{2}(f, g, \rho, b)(n, \tau)=\frac{1}{\left(1+\left|\tau-n^{2}\right|\right)^{1-b}(1+|n|)^{\rho}} \\
\times \sum_{n_{1}} \int \frac{f\left(n_{1}, \tau_{1}\right)\left(1+\left|n_{1}\right|\right)^{\rho}}{\left(1+\left|\tau_{1}+n_{1}^{2}\right|\right)^{b}} \frac{g\left(n-n_{1}, \tau-\tau_{1}\right)\left(1+\left|n-n_{1}\right|\right)^{\rho}}{\left(1+\left|\tau-\tau_{1}-\left(n-n_{1}\right)^{2}\right|\right)^{b}} d \tau
\end{gathered}
$$

the estimate

$$
\left\|\mathcal{B}_{2}(f, g, \rho, b)\right\|_{\ell_{n}^{2} L_{\tau}^{2}} \leq C\|f\|_{\ell_{n}^{2} L_{\tau}^{2}}\|q\|_{\ell_{n}^{2} L_{\tau}^{2}}
$$

fails. For $N \in \mathbb{Z}^{+}$define $f_{N}(n, \tau)$ as in (5.46) and

$$
g_{N}(n, \tau)=b_{n} \chi\left(\left(\tau-n^{2}\right) / 2\right), \quad \text { with } \quad b_{n}= \begin{cases}1 & n=-N, \\ 0, & \text { elsewhere }\end{cases}
$$

Using that

$$
a_{n_{1}} b_{n-n_{1}} \neq 0 \text { if and only if } n_{1}=N \text { and } n=0
$$

and

$$
\int \chi\left(\left(\tau_{1}+n_{1}^{2}\right) / 2\right) \chi\left(\tau-\tau_{1}-\left(n-n_{1}\right)^{2} / 2\right) d \tau \cong \chi\left(\tau-\left(n-n_{1}\right)^{2}+n_{1}^{2}\right) \cong \chi(\tau)
$$

in (5.56) we obtain

$$
\mathcal{B}_{2}\left(f_{N}, g_{N}, \rho, b\right)(0, \tau) \geq \frac{c N^{2 \rho}}{(1+|\tau|)^{1-b}} \chi(\tau)=c N^{2 \rho} \chi(\tau) .
$$

Finally from (5.57) we obtain

$$
N^{2 \rho} \leq c
$$

\section{REFERENCES}

[BKPSV] B. Birnir, C. E. Kenig, G. Ponce, N. Svanstedt and L. Vega, On the ill-posedness of the IVP for the generalized Korteweg-de Vries and nonlinear Schrödinger equations, J. London Math. Soc. (to appear).

[B] J. Bourgain, Fourier restriction phenomena for certain lattice subsets and applications to nonlinear evolution equations, Geometric and Functional Anal. 3 (1993), 107-156, 209-262. MR 95d:35160b

[C] T. Cazenave, An introduction to nonlinear Schrödinger equations, Textos de Métodos Matemáticos 22 Universidade Federal do Rio de Janeiro.

[CW] T. Cazenave and F. Weissler, The Cauchy problem for the critical nonlinear Schrödinger equation in $H^{s}$, Nonlinear Anal. TMA 14 (1990), 807-836. MR 91j:35252

[D] D. Dix, Nonuniqueness and uniqueness in the initial-value problem for Burger's equation, SIAM J. Math. Anal. (to appear).

[F1] C. Fefferman, Inequalties for strongly singular convolution operators, Acta Math. 124 (1970), 9-36. MR 41:2468

[F2] C. Fefferman, A note on spherical summation multipliers, Israel J. Math. 15 (1973), 44-52. MR 47:9160

[GV1] J. Ginibre and G. Velo, On a class of nonlinear Schrödinger equations, J. Funct. Anal. 32 (1979), 1-71. MR 82c:35057 and MR 82c:35058

[GV2] J. Ginibre and G. Velo, Scattering theory in the energy space for a class of nonlinear Schrödinger equations, J. Math. Pure Appl. 64 (1985), 363-401. MR 87i:35171

[K1] T. Kato, Quasi-linear equations of evolutions, with applications to partial differential equations, Lecture Notes in Math, 448, Springer-Verlag, 1975, pp. 27-50. MR 53:11252 
[K2] T. Kato, On the Cauchy problem for the (generalized) Korteweg-de Vries equation, Advances in Math. Supp. Studies, Studies in Applied Math. 8 (1983), 93-128. MR 86f:35160

[K3] T. Kato, Nonlinear Schrödinger equation, Schrödinger operators, Lecture Notes in Physics, 345 (H. Holden and A. Jensen, eds.), Springer-Verlag, 1989, pp. 218-263. MR 91d:35202

[KPV1] C. E. Kenig, G. Ponce and L. Vega, Well-posedness and scattering results for the generalized Koreteweg-de Vries equation via the contraction principle, Comm. Pure Appl. Math. 46 (1993), 527-620. MR 94h:35229

[KPV2] C. E. Kenig, G. Ponce and L. Vega, The Cauchy problem for the Korteweg-de Vries equation in Sobolev spaces of negative indices, Duke Math. J. 71 (1993), 1-21. MR 94g:35196

[KPV3] C. E. Kenig, G. Ponce and L. Vega, Bilinear estimates with applications to the KdV equations, J. Amer. Math. Soc. (to appear).

[KM1] S. Klainerman and M. Machedon, Space-time estimates for null forms and the local existence theorem, Comm. Pure Appl. Math. 46 (1993), 1221-1268. MR 94h:35137

[KM2] S. Klainerman and M. Machedon, Smoothing estimates for null forms and applications, preprint.

[L] H. Lindblad, A sharp counter example to local existence of low regularity solutions to nonlinear wave equations, Duke Math. J. 72 (1993), 503-539. MR 94h:35165

[PS] G. Ponce and T. Sideris, Local regularity of nonlinear wave equations in three space dimensions, Comm. P.D.E. 18 (1993), 169-177. MR 95a:35092

[S] R. Strichartz, Restriction of Fourier transforms to quadratic surface and decay of solutions of wave equations, Duke Math. J. 44 (1977), 705-714. MR 58:23577

[T] Y. Tsutsumi, $L^{2}$-solutions for nonlinear Schrödinger equations and nonlinear groups, Funk. Ekva. 30 (1987), 115-125. MR 89c:35143

[Z] A. Zygmund, On Fourier coefficients and transforms of functions of two variables, Studia Math. 50 (1974), 189-201. MR 52:8788

Department of Mathematics, University of Chicago, Chicago, Illinois 60637

E-mail address: cek@math.uchicago.edu

Department of Mathematics, University of California, Santa Barbara, California 93106

E-mail address: ponce@math.ucsb.edu

Departamento de Matematicas, Universidad del Pais Vasco, Apartado 644, 48080 BilBAO, SPAIN

E-mail address: MTPVEGOL@lg.ehu.es 